Title:

Author(s):

Submitted to:

Photoemission in Strongly Correlated Crystalline f-Electron Systems: A need for a new approach

$$
\text { CONF-9806153-- }
$$
A.J. Arko
J.J. Joyce
J.L. Sarrao
Z. Fisk
J.L. Smith
J.D. Thompson
M.F. Hundley
A. Menovsky
A. Tahvildar-Zadeh
M.A. Jarrell

MASTER

International Workshop on Electron Correlations and Materials Properties
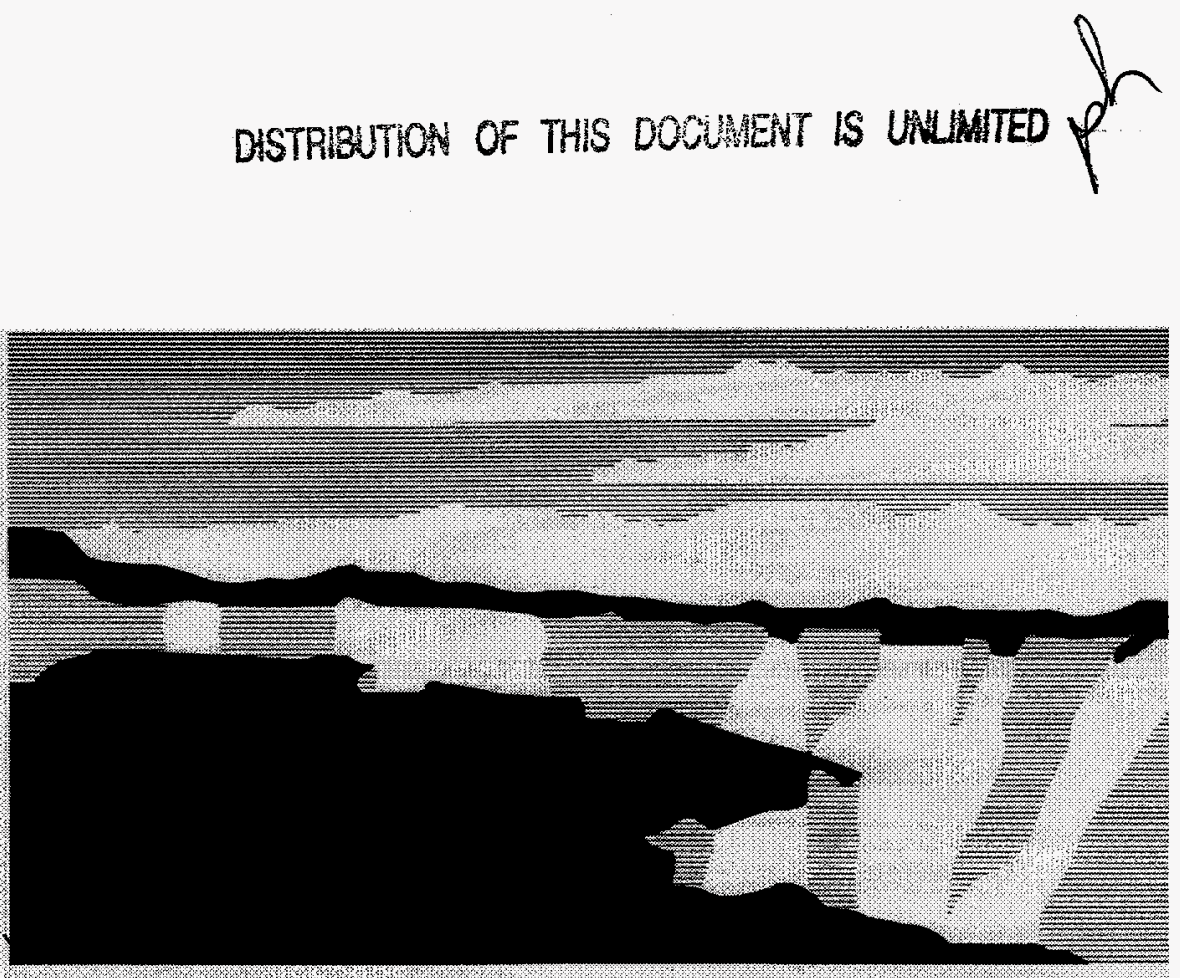

Los Alamos National Laboratory, an affirmative action/equal opportunity employer, is operated by the University of California for the U.S. Department of Energy under contract W-7405-ENG-36. By acceptance of this article, the publisher recognizes that the U.S. Government retains a nonexclusive, royalty-free license to publish or reproduce the published form of this contribution, or to allow others to do so, for U.S. Government purposes. The Los Alamos National Laboratory requests that the publisher identify this article as work performed under the auspices of the U.S. Department of Energy. 


\section{DISCLAIMER}

This report was prepared as an account of work sponsored by an agency of the United States Govemment. Neither the United States Government nor any agency thereof, nor any of their employees, makes any warranty, express or implied, or assumes any legal liability or responsibility for the accuracy, completeness, or usefulness of any information, apparatus, product, or process disclosed. or represents that its use would not infringe privately owned rights. Reference herein to any specific commercial product, process, or service by trade name, trademark, manufacturet, or otherwise does not necessarily constitute or imply its endorsement, recommendation, or favoring by the United States Government or any agency thereof. The views and opinions of authors expressed herein do not necessarily state or reflect those of the United States Government or any agency thereof. 


\section{DISCLAIMER}

Portions of this document may be illegible in electronic image products. Images are produced from the best available original document. 


\title{
PHOTOEMISSION IN STRONGLY CORRELATED CRYSTALLINE f-ELECTRON SYSTEMS: A NEED FOR A NEW APPROACH
}

\author{
A.J. Arko', J.J. Joyce ${ }^{1}$, J. Sarrao ${ }^{1}$, Z. Fisk', J.L. Smith ${ }^{1}$, J.D. Thompson ${ }^{1}$, \\ M. Hundley ${ }^{1}$, A. Menovsky ${ }^{2}$, A. Tahvildar-Zadeh ${ }^{3}$, and M.A. Jarrell ${ }^{3}$ \\ ${ }^{1}$ Los Alamos National Laboratory \\ Los Alamos, Nm 87545 \\ ${ }^{2}$ Natuurkundig Laboratorium \\ University of Amsterdam \\ The Netherlands \\ ${ }^{3}$ Univ of Cincinnati \\ Cincinnati, Ohio 45221-0011
}

\section{INTRODUCTION}

The unusual properties of heavy fermion (or heavy electron) materials have sparked an avalanche of research over the last two decades in order to understand the basic phenomena responsible for these properties. The reader is referred to numerous review articles, such as Fisk et. al., (1995), Grewe and Steglich (1991), Hess et. al., (1993), Lawrence and Mills (1991), Ott and Fisk (1987), Stewart (1984), Thompson and Lawrence (1994). The term heavy fermion refers to materials (thus far only compounds with elements having an unfilled $4 f$ or $5 f$ shells) whose electronic properties suggest that the conduction electrons have a very heavy effective mass. This is irferred from the linear term in the low specific heat $(\mathrm{Cp}=\gamma \mathrm{T}+$ $O T^{2}$ ) which usually has an upturn at low temperatures and suggests [some of the first discoveries were by Andres et. al. (1975), followed by Steglich (1979), Stewart et. al (1984), Ott et. al. (1983)| $\gamma$-values as large as $1500 \mathrm{~mJ} / \mathrm{mole}^{2} \mathrm{~K}^{2}$. deHaas-van Alphen measurements have contirmed the existence of heavy masses [Springford and Reinders (1988), Lonzarich (1988), Onuki (1993)]. The quadratic term in the resistivity and the Pauli susceptibility also indicate the existence of a mass enhancement. It is well established that indeed it is the $f$ clectrons that are responsible for the unusual properties [Grewe and Steglich (1991)].

Magnetic susceptibility measurements, $\chi$, generally yield a Curie-Weiss behavior at high temperatures with a well developed moment [see, for example, Thompson and Lawrence (1994)], which would be consistent with a localized behavior of the f-electrons. Indeed, the [-clectrons appear to behave as non-interacting single impurities at elevated temperature, while below some characteristic temperature, usually referred to as the Kondo Temperature or $T_{K}$, the susceptibility levels off or even decreases. This phenomenon is interpreted as a compensation of the magnetic moment by the ligand conduction electrons that are believed to align anti-parallel to the f-electrons to form a singlet state [see, for example, Grewe and Steglich (1991)]. At still lower temperatures a dramatic drop in the electrical resistivity, $\rho$, is associated with the formation of a coherent periodic lattice of these compensated f-electrons so that the low temperature electronic structure can be consistently interpreted as being due to a band of very heavy mobile fermions. This is of ten referred to as the Kondo lattice or the Anderson lattice. The concept of a Kondo or Anderson lattice appcars to date back to Lawrence et. al. (1981), but the reader is referred also to an excellent review of theorctical approaches by Lee et. al. (1986). 
There is, however, another school of thought that claims that the f-electrons form well-defined Bloch states and very narrow bands at all temperatures [Zwicknagl (1992), Liu (1993, 1997). Shong and Cooper (1995)]. While there is little quarrel with this view at low temperatures contentional band theory (using the Local Density Approximation, or LDA) is clearly unable to explain the high-temperature properties as well as the very heavy mass. Renormalized band theory [Strange and Newns (1986), Zwicknagl (1992)] may yet prove useful, cspccially those that incorporate physics similar to the Kondo effect and its temperature dependence, perturbatively [Steiner (1994), Norman (1985), Norman et. al. (1985)]. However, the apparent success of the Single Impurity Model (SIM) in explaining macroscopic bulk phenomena suggests a correctness, at least at some level, of the model, though recent results ( $\mathrm{C}$. Booth, private communication) indicate that attempts to extract bandwidths from bulk data yicld totally unphysical results. This suggests a need to re-evaluate the SIM even in connection with bulk data. The heart of the issue clearly then is the exact role of the fclectrons in the formation of the heavy fermion state. In this respect the effect of the periodic lattice is non-negligible. The periodic Anderson model (PAM) has made headway in treating this problem and will be discussed in detail though other periodic models are not yet ruled out.

As stated, the localized approach utilizes (SIM) as the starting point. The assumption here is that even in periodic systems (i.e., ordered compounds), where an f-electron exists within each unit cell, the $\mathrm{f}-\mathrm{f}$ overlap is so small that at high temperatures the f-electrons can be treated as completely localized impurities in a sea of ligand conduction electrons having a density of states $N(E)$. It is the very slight residual hybridization with $N(E)$ which yields the low temperature behavior as the hybridization energy exceeds $\mathrm{k}_{\mathrm{B}} \mathrm{T}$. The Gunnarsson and Schonhammer $(1985,1986)$ approximate solution of SIM calculates the particle-hole excitation spectrum (we will refer to it as GS) and has been hailed as a breakthrough in the understanding of heavy fermion systems. The non-crossing approximation, or NCA, has in addition been able to introduce [Bickers (1987), Bickers et. al. (1985 and 1987)] the effects of temperature, and has recently included the effect of finite $f-f$ Coulomb repulsion, $U_{\text {ff }}$, [Pruschke (1989)] thus including the possibility of double occupancy of f-levels. Without a doubt the GS and NCA approximations are the most comprehensive, as well as the most widely accepted approximations of the particle-hole excitation spectrum in heavy fermions, in the Kondo limit. More recently numerical renormaliztion group (NRG) [Wilson (1975)] and Quantum Monte-Carlo (QMC) [Hirsch and Fye (1986)] approaches have been extended to treat dynamics [Oliveira and Wilkins (1981), Silver et. al. (1990), Jarrell and Gubernatis (1996)]. A universal behavior for the f-electron DOS comes out of these approximate solutions, the core of which is the prediction of scaling with $T_{K}$.

Photoelectron spectroscopy (often referred to as PES in the following sections), the most direct measurement of the electronic structure of a material, should in principle be able to shed considerable light on this matter. In general the distinction between a localized and a band-like state is trivially observed in band dispersion. However, in the case of heavy fermions such distinctions pose a serious problem since any dispersion of an $\mathrm{f}$-band within the coherent state is expected to be of the order of $T_{K}$ which is generally far below the resolution of present day apparatii. Nonetheless, PES measurements on heavy fermions have been abundant [For a review of some of the early work on poly-crystals see Allen et. al. (1986 and 1992)]. (W ing to the very specific predictions of the GS and NCA approximations regarding the width, position, spectral weight, and temperature dependence of the f-electron DOS, which lends itself readily to a photoemission measurement.

Much of the past work was performed on poly-crystalline samples, scraped in-situ to expose a clean surface for PES. Further, despite the fact that the features studied were at least an order of magnitude narrower than the then-available experimental resolution (or perhaps because of it), good agreement with the GS approximation was of ten reported [Patthey et. al. (1985, 1986, 1987a and b, 1990), Malterre et. al. (1992a and b), Tjeng et. al. (1993), Kang et. al. (1990), Allen et. al. (1986), Liu et. al. (1992), Weibel et. al. (1993)]. There have since been considerable advances both in the quality of specimens as well as experimental resolution, which raise questions regarding these conclusions. Much of the past work on poly-crystalline samples has been reported in several review articles, most notably Allen et. al $(1986,1992)$, and it is not necessary here to review those efforts again, with the exception of subsequent work performed at high resolution. The primary focus of the present review will be on new measurements obtained on single crystals, cleaved or prepared in situ and measured at high resolution, which seem to suggest [Joyce et. al. (1992a,b,c), (1993a,b), 
(1994a, b), (1996), Arko et. al. (1993), (1994), Andrews et. al. (1994), (1995a,b), (1996), Blyth et. al. (1992), (1993)] that agreement with the GS and NCA approximations is less than perfect, and that perhaps the starting models need to be modified, or that even an entirely new approach is called for. Of the promising new models the Periodic Anderson Model is most closely related to the SIM. Indeed, at high temperatures it reverts to the SIM. Howcver, the charge polaron model of Liu (1997) as well as the two-electron band model of Sheng and C(x)per (1995) cannot yet be ruled out.

Inasmuch as the bulk of the single crystal work was performed by our own Los Alamos group [Andrews et. al. (1995a,b), (1996), Joyce et. al, (1996)], this review will draw heavily on those results. Moreover, since the GS and NCA approximations represent the most comprehensive and widely accepted treatment of heavy fermion PES, it is only natural that we primarily concern ourselves with analysis of PES data in terms of these models, in order to thoroughly test their validity in light of the new data.

The evolution of theoretical models has been driven by experiment. To date, the f component of the one electron addition/removal spectra of alloys containing Ce ions, as wcll as ordered $\mathrm{Ce}$ compounds, are qualitatively similar. Due to the difficulty of separating out the f component of the spectrum, the only concentration dependence that has been identified is the linear dependence of the overall intensity. This unsatisfactory state of affairs has caused theorists to focus their attentions primarily on single impurity models that have the same qualitative features as observed in experiments. Thereby, effects that are present in ordered compounds have been, hitherto, largely ignored. In this review our main focus will be on ordered compounds, indeed, single crystals.

\section{THEORETICAL CONSIDERATIONS}

\section{Kondo Models}

Most models are based on the single impurity Anderson (1961) model, which describes an $\mathrm{f}$ shell that is bathed in a Fermi-sea of conduction electrons. The Hamiltonian, $\mathrm{H}$, can be written as the sum of three terms,

$$
\mathrm{H}=\mathrm{H}_{\mathrm{f}}+\mathrm{H}_{\mathrm{d}}+\mathrm{H}_{\mathrm{fd}}
$$

where $\mathrm{H}_{\mathrm{f}}$ describes the $\mathrm{f}$ electron system, $\mathrm{H}_{\mathrm{d}}$ describes the conduction electron systems and $\mathrm{H}_{\mathrm{fd}}$ describes the coupling between these two systems. The Hamiltonian $\mathrm{H}_{\mathrm{f}}$ can be written as

$$
\mathrm{H}_{\mathrm{f}}=\sum \mathrm{E}_{\mathrm{f}} \mathrm{f}_{\alpha}^{+} \mathrm{f}_{\alpha}+\sum \mathrm{U}_{\mathrm{ff}} / 2 \mathrm{f}_{\alpha}^{+} \mathrm{f}_{\beta}^{+} \mathrm{f}_{\beta} \mathrm{f}_{\alpha}
$$

where the operators $\mathrm{f}_{\alpha}^{+}$and $\mathrm{f}_{\alpha}$ respectively create and annihilate an electron of spin $\alpha$ in the $f$ shell. The first term, proportional to $E_{f}$, represents the binding energy of an electron in the $f$ level, and the second term represents the coulomb repulsion between pairs of electrons in the f shell. The Pauli exclusion principle makes this term, proportional to $U_{\mathrm{ff}}$, inactive when $\alpha=$ $\beta$. The conduction electron states are described by $\mathrm{H}_{\mathrm{d}}$, as non-interacting conduction electrons in band states,

$\mathrm{H}_{\mathrm{d}}=\sum \mathrm{e}(\underline{\mathrm{k}}) \mathrm{d}^{+}{ }_{\mathrm{k}}, \alpha, \mathrm{d}_{\mathrm{k}}, \alpha$
where the operators $\mathrm{d}^{+} \mathrm{k}, \alpha$ and $\mathrm{d}_{\mathrm{k}, \alpha}$, respectively, create and annihilate electrons of spin $\alpha$ in the conduction band state labeled by the Bloch wave-vector $\underline{k}$. The quantity $e(\underline{k})$ is the dispersion relation of the conduction band states. The $f$ electron and $\mathrm{d}$ electron systems are couplad ia the hybridization process, $\mathrm{H}_{\mathrm{fd}}$, which can be written as

$$
\mathrm{H}_{\mathrm{fd}}=\sum\left(\mathrm{V}(\underline{\mathrm{k}}) \mathrm{d}^{+} \mathrm{k}, \alpha \mathrm{f}_{\alpha}+\mathrm{V}^{*}(\underline{\mathrm{k}}) \mathrm{f}^{+} \alpha \mathrm{d}_{\underline{\mathrm{k}}, \alpha}\right)
$$

The first term represents a process in which an electron of spin $\alpha$ is taken out of the $f$ shell and placed in a conduction band state, the Hermitean conjugate represents the reverse proccss. The hybridization term conserves the spin index $\alpha$. This term, when treated in second grder perturbation theory yields a lifetime, or effective width of the flevel given by $\Delta=\pi / \mathrm{V}$ $F^{2} \rho(\mu)$, where $\rho(\mu)$ is the conduction band density of states at the fermi-level.

The solutions to the above Hamiltonians are lengthy and laborious. Gunnarsson and Schonhammer $(1985,1986)$ after a series of approximations succeeded in calculating the particle-hole excitation spectrum for the case of $T=0$. Subsequently, Bickers, Cox and Wilkins (1985, 1987), using the non-crossing approximation (NCA) succeeded in calculating the spectrum at finite temperatures, but at infinite $U_{\mathrm{ff}}$. A finite $U_{\mathrm{ff}}$ calculation was eventually obtained by Pruschke (1989). It is beyond the scope of this paper, however, to review in detail the theorctical approaches. Rather it is the comparison of theory to experiment, and the 
possible sorting out of the models which attracts our attention. For this reason we will rather show the excitation spectrum predicted by NCA since the comparison to NCA will form the bulk of this paper. The inconsistencies encountered between experiment and NCA predictions will force us to consider other models, especially the periodic Anderson Model.

Nozicres (1985) has pointed out that the formation of the polarization clouds may be expected to only involve the conduction electrons within $\mathrm{k}_{\mathrm{B}} \mathrm{T}_{\mathrm{k}}$ of the fermi-energy. Thus the number of electrons forming the compensating polarization cloud may be expected to be of the order of $k_{13} T_{k} \rho(\mu)$, where $\rho(\mu)$ is the conduction band density of states evaluated at the fermi-level. Since $k_{B} T_{k}$ is much less than the depth of the occupied portion of the conduction band density of states, this yields an estimate of much less than one conduction electron per f ion. Thus, the estimated number of electrons in the polarization cloud is less than the number of $\mathrm{f}$ moments to be screened so an altemative description, other than the single impurity description, may have to be utilized for concentrated compounds. The periodic Anderson Model (PAM) appears to fit this description since in this picture the compensationg conduction electrons are allowed to hop from f-site to f-site thus effectively screening in spite of insufficient numbers, and forming a narrow hybridized f-band - a magnetic polaron. .

But before considering this approach, let us look at the NCA predictions. A graphic representation of the predictions of NCA is perhaps most useful to the reader. An unbroadened calculated spectrum is shown in Fig. la (code provided by D.L. Cox) for a typical heavy fermion material having a $T_{K}$ of $35 \mathrm{~K}$, a model (flat) conduction band $5 \mathrm{eV}$ wide, crystal field levels at -25 and $-48 \mathrm{meV}, \mathrm{U}_{\mathrm{f}}$ of $7 \mathrm{eV}$, and a bare f-level positioned at $\varepsilon_{\mathrm{f}} \approx-2.3 \mathrm{eV}$. These parameters approximately correspond to those of the heavy fermion compound $\mathrm{CeSi}_{2}$ which has been heavily reported in literature [Patthey et. al (1986), Joyce et. al. (1992c), Takahashi et. al. (1994), Malterre et. al. (1992a,b), Garnier et al. (1997)]. It should be stressed that within the SIM the 4 f DOS in Fig. Ia is obtained by averaging the hybridization : :

Fig. 1 (a) Particle-hole excitation spectrum calculated for $\mathrm{T}=11 \mathrm{~K}$ using NCA code provided by D.I. Cox. Parameters approximately correspond to those expected for $\mathrm{CeSi}_{2}$, namely: $T_{K}=37 \mathrm{~K}, W=5 \mathrm{eV}$ (Lorentzian), $\Gamma=0.1+39, \Delta_{\mathrm{s}}=280 \mathrm{meV}, \mathrm{e}_{i}=-2.4 \mathrm{eV}$, $\Delta_{\mathrm{CF} 1}=-25 \mathrm{mel}, \Delta_{\mathrm{CF} 2}=-18 \mathrm{eV}, \mathrm{L}_{\text {if }}=\infty$. (b) Blow-up of near- $\mathrm{E}_{\mathrm{F}}$ region. Dark line corresponds to convolution of spectrum with a $T=0 \mathrm{~K}$ Fermi function. (c) Calculated PES spectrum at $\mathrm{T} \approx 0 \mathrm{~K}$ using three different instrument resolutions. 
over all conduction electron states, so that dispersion is precluded. To be more precise, we are not actually dealing with a 4 f DOS at the Fermi energy but rather only the response of the system to a corc hole. We will nevertheless refer to the intensity at the Fermi energy as the $4 f$ DOS. This was nicely explained for the experimentalist by Huffner (1986) and Huffner and Schlapbach (1986). In any case it is argued that NCA yields an excellent representation of the 4f DOS at high temperatures, while at low temperatures (far below $T_{K}$ where a Kondo Lattice model is more appropriate) any dispersion in the coherent state is in any case smaller than $k_{B} T_{K}$ and thus negligible. The essential predictions of SIM (i.e., the scaling of $4 f$ spectral fcatures with $T_{K}$ ) are of necessity retained at all temperatures.

Although Ce contains only one f-electron, the GS theory [Gunnarsson and Schonhammer (1985, 1986)] as well as NCA [Bickers et. al. (1985, 1987)] predicts at least three features in a PES spectrum (occupied states) and at least two features in a BIS spectrum (romaining 13 empty states). In Fig. 1a the occupied features are labeled as the $\mathrm{f}^{0}$ or "main" pcak which represents the primary $f^{i}$ to $f^{0}$ excitation, while the $f^{1}$ portion of the spectrum consists of the narrow $4 f_{5 / 2}$ feature (called the Kondo Resonance, $K R$ ) located at $k_{B} T_{K}$ above $E_{F}$ with only a small occupied tail extending below $E_{F}$ (see Fig. 1b), as well as a spin-orbit split sideband (the $4 \mathrm{f}_{7 / 2}$ feature) located at $\Delta_{\mathrm{so}}-\mathrm{T}_{\text {so }}$ below the $\mathrm{KR}$ (Here $\Delta_{\text {so }}$ is the spin-orbit splitting while $T_{s o}$ is an effective Kondo temperature for the sideband, usually about an order of magnitude larger than $T_{K}$ ). Above the Fermi energy we have the bulk of the $K R$ at $\approx k_{B} T_{K}$, an equally intense spin-orbit sideband at $+\Delta_{\mathrm{SO}}$, as well as a broad $\mathrm{f}^{2}$ feature (not shown) corresponding to double f-occupancy at finite $U_{\mathrm{ff}}$, and situated at $U_{\mathrm{ff}}$ above the occupied $\mathrm{f}^{\circ}$ peak. The KR may additionally be split into crystal field levels situated at $\Delta_{C F}$ relative to $T_{K}$, which have their own effective Kondo temperature, $T_{C F}$, and which may actually carry more spectral weight than the KR. These are shown in the expanded view in Fig. $1 \mathrm{~b}$.

Because of particle-hole symmetry, the PES spectrum for Yb heavy fermions, where one has only one $\mathrm{f}$-hole $\left(\mathrm{f}^{13}\right.$ vs $\left.\mathrm{f}^{14}\right)$ rather than one $\mathrm{f}$-electron, can be obtained from Fig. la simply by applying mirror symmetry about the Fermi energy [Bickers et. al. (1987)]. Thus in $\mathrm{Yb}$ heavy fermions the bulk of the KR is predicted to be occupied which makes them ideal candidates for studying spectral weights, widths, and temperature dependencies. The ability to determine $\mathrm{n}_{\mathrm{f}}$, the $\mathrm{f}$-hole occupancy (f-electron for Ce compounds), is likewise made possible in Yb compounds since the f-spectral weight is fully occupied. The $\mathrm{n}_{\mathrm{f}}$ more or less corresponds to the percentage of the $\mathrm{f}$-hole in the $\mathrm{f}^{13}$ state, which should be close to unity in the Kondo limit. The $\mathrm{f}^{1+}$ spectral weight in the Kondo limit is then roughly $\left(1-n_{f}\right)$ with small adjustments for the differences in the degeneracy

From Bickers et. al. (1987) we note several important points regarding Fig. 1a. (1) The width of the $f^{\circ}$ peak varies with the hybridization $\Gamma$ (width $=28 \Delta$ when using a model DOS), and has a spectral weight of about one electron (i.e., $n_{f} \approx 1$ ). The width of the $K R \approx$ $k_{B} T_{K}$, while its total spectral weight (including the unoccupied portion) scales with $T_{K}$ approximately as $\pi T_{K} / \Gamma$. The position of the SO sideband varies as $\Delta_{S O}-T_{\text {so }}$ so that for increasing $T_{K}$ it shifts closer to $E_{F}$ as $T_{\text {so }}$ also increases along with $T_{K}$. Thus most of the spectral features at $E_{F}$ increase with $T_{K}$ (the sidebands, of course, scale directly with $T_{S o}$ and $T_{C F}$ ) and readily lend themselves to measurement in PES,

The cxistence of crystal field levels yields additional features near the Fermi energy. For the spectrum of Fig. la two crystal field levels have been introduced (at $-24 \mathrm{meV}$ and -48 meV) 4 approximately correspond to those reported for $\mathrm{CeSi}_{2}$ in neutron diffraction measurements [Galera et. al., (1989)]. A blow up of the near- $E_{\mathrm{F}}$ region is shown in Fig. 1b with the crystal field levels delineated. Note that on the occupied portion of the spectrum these levels are relatively weak and in fact do not account for the bulk of the occupied spectral weight. Although crystal field levels have never been unambiguously observed in a PES measurement, their theoretical spectral weight cannot always be assumed to be small (it depends on the parameters), particularly in $\mathrm{Yb}$ compounds.

In an actual PES measurement the spectrum in Fig. 1a would be convoluted with a Fermi function to yield only the occupied states, as well as broadened by instrument resolution. The dark lined spectrum in Fig. $1 \mathrm{~b}$ corresponds to a convolution with a $\mathrm{T}=0 \mathrm{~K}$ Fermi function. In Fig. Ic this $T=0 \mathrm{~K}$ spectrum has been further broadened with a $20 \mathrm{meV}$ Gaussian to match the stated resolution of Patthey et. al. (1987a) as well as with an $80 \mathrm{meV}$ Gaussian to match the best resolution of a measurement at resonance ( $\mathrm{hv}=120 \mathrm{eV}$ ). This then is the expected shape of a typical Ce heavy fermion PES spectrum, while a typical Yb spectrum would mimic the unoccupied states of Fig. la broadened by instrument resolution. 
Besides the scaling with $T_{K}$, a major prediction of SIM and its extensions (in particular, NCA) is the temperature dependence of the KR as shown by Bickers et. al. (1987). They showed that a substantial decrease in the $\mathrm{KR}$ intensity already exists at $0.23 \mathrm{~T}_{\mathrm{K}}$, while at $29 \mathrm{~T}_{\mathrm{K}}$ all the $f^{\prime}$ spectral features have been renormalized to nearly zero. A real spectrum at temperatures comparable to $T_{K}$ may be complicated somewhat by the existence of possible crystal field (or CF) levels where $\mathrm{T}_{\mathrm{K}}<\mathrm{T}_{\mathrm{CF}}<\mathrm{T}_{\mathrm{SO}}$. Nonetheless, at $29 \mathrm{~T}_{\mathrm{K}}$ all $\mathrm{f}^{1}$ states should renormalize to near $7 \mathrm{cr}$.

Effects of CF levels and finite $U_{\text {ff }}$ have been singled out as complicating the PES spectra of heary fermions [Allen et. al., 1986, 1992]. In particular, because of their larger cffective Kondo temperature, CF levels may result in a lack of scaling with $T_{K}$. (Huffner (1992) view's these as single particle states, although that argument cannot not hold for large$\mathrm{T}_{\mathrm{K}}$ materials such as $\mathrm{CeBe}_{13}$ where the width of the $\mathrm{KR}$ is theoretically broader than any $\mathrm{CF}$ states). Likewise, it is possible that spectral weight from the very broad $f^{2}$ states at finite $U_{\text {ff }}$ may spill over into the occupied portion of the spectrum and strongly affect the scaling. Joyce and Arko (1993a) have shown however that while CF states indeed add extra weight (though not much), the scaling with $\mathrm{T}_{K}$ remains dominant within GS theory.

The effect (or lack thereof) of the finite $U_{\mathrm{ff}}$ was also demonstrated by Joyce and Arko (1993a). Space limitations prevent extensive discussion Its net effect is merely to increase all intensities without changing the overall shape of the spectra, thus keeping all relative weights unaffected. The lack of an effect is not surprising since spillover of the very broad $f^{2}$ states into the occupied portion of the spectrum should resemble the extreme end of the tail of a Gaussian which cannot affect the $f^{1}$ features much more than the $f^{0}$ peak.

In summary, the important predictions of GS and NCA are those of scaling of $4 f$ features with hybridization (and hence $T_{K}$ ) together with a strong temperature dependence of these features as the hybridization decreases with increasing temperature. The scaling is: complicated somewhat by the exact choice of parameters, but the increase in the $\mathrm{f}^{1}$ spectral weight with $T_{K}$ is at the very least monotonic.

\section{Periodic Models}

We will see that PES results from single crystals fail to reproduce the NCA predicted spectra, differing qualitatively and not just quantitatively. In general there is much less temperature than predicted, while $n_{\mathrm{f}}$ values, especially in $\mathrm{Yb}$ compounds, are dramatically too low (i.e., too much spectral weight in the $\mathrm{f}^{14}$ peaks). Most importantly, dispersion is of ten observed in f-electron features at very high temperatures relative to $T_{K}$ (i.e., at temperatures where the susceptibility displays Curie-Weiss behavior), belying the concept of a single impurity.

While several theoretical approaches [Tahvildar-Zadeh, 1997,1998, Zwicknagel, 1992, Steiner et al., 1994, Liu, 1993, 1997, Shenh and Cooper, 1995] hold promise of accounting for dispersive behavior, we focus below on the Periodic Anderson Model [Tahvildar-Zadeh, 1997], PAM, which in its preliminary stages appears to capture the essential physics. Furthermore, it represents merely an extension of the SIM to periodic systems and should thus be most pallatable to proponents of the SIM. Quite possibly these different approaches, mostly based on the Anderson Hamiltonian, may eventually converge on the vame tinal result, namely some form of renormalized bands [Zwicknagel, 1992, Steiner et al. 1944 | displaying minimal temperature dependence. A necessary flattening of these bands at $E_{1}$ " ith temperature, due to the correlations, yields the heavy electron masses well as a mimicing, in parts of the Brillouin zone, of the feature called the Kondo resonance.

The PAM is believed to most correctly describe the strong correlation of electrons in Kondo lattice systems; i.e., stoichiometric compounds with $4 f$ or $5 f$ electrons in the valence shell. While for more than a decade the SIM has been the paradigm for comparison with PES, it cannot account for the coherent nature of electrons (i.e., periodic Bloch states) now observed both above and below $\mathrm{T}_{\mathrm{K}}$. The PAM accounts for these effects. Unfortunately, the difficult nature of PAM calculations necessitates the use of simple generic models rather than real systems. Indeed, the calculation is done in intinite dimensions. Nevertheless the predictad trends hold the promise of much better agreement with PES data.

The PAM Hamiltonian on a D-dimensional hypercubic lattice is, 


$$
\begin{aligned}
& H=\frac{-t^{*}}{2 \sqrt{D}} \sum_{i, j, \sigma}\left(d_{i \sigma}^{\dagger} d_{j \sigma}+\text { h.c. }\right) \\
& +\sum_{i \sigma}\left(\varepsilon_{d} d_{i \sigma}^{\dagger} d_{i \sigma}+\varepsilon_{f} f_{i \sigma}^{\dagger} f_{i \sigma}\right)+V \sum_{i \sigma}\left(d_{i \sigma}^{\dagger} f_{i \sigma}+\text { h.c. }\right) \\
& +\sum_{i} U\left(n_{f i \uparrow}-1 / 2\right)\left(n_{f i \downarrow}-1 / 2\right) .
\end{aligned}
$$

In the above equation, $d(f)_{i \sigma}^{\prime \prime}$ destroys (creates) a $d(f)$-electron with spin s on site $\mathrm{i}$. The hopping is restricted to the nearest neighbors and scaled as $t=t^{*} / 2 \sqrt{D}$. Here $\mathrm{t}^{*}=1$, the width of the Gaussian density of states, as the energy scale. The unhybridized d-bandwidth is $2 \sqrt{D}$, and this broadens further with hybridization. $U$ is the screened on-site Coulomb repulsion for the localized $\mathrm{f}$-states and $\mathrm{V}$ is the hybridization between $\mathrm{d}$-and $\mathrm{f}$-states. This model, then, retains the screening and moment formation of the impurity problem, but is further complicated by the lattice effects and the interaction between the moments.

Metzner and Vollhardt [1989] observed that the irreducible self-energy and the vertex functions become purely local as the coordination number of the lattice increases. As a consequence, the solution of this interacting lattice model may be mapped onto the solution of a local correlated impurity coupled to an effective bath which is self-consistently determined. [Pruschke et al., 1996]. The Quantum Monte Carlo (QMC) algorithm of Hirsch and Fye [1989] is employed to solve the remaining impurity problem. The basic outputs of this procedure are the $f$ and $d$ Green's functions of the model in Matsubara frequency. The maximum entropy method (MEM) is then employed to analytically continue these functions to real frequency [Jarrell et al., 1996].

Initial results indicate a substantial differences between the PAM and the SIM approaches, especially in the much slower temperature dependence of the PAM. While both yield a sharp peaking of the DOS at $E_{F}$ that can be interpreted as a Kondo resonance, thePAM calculations find that these are narrow bands both above and below $T_{K}$, as shown in Fig. 2. Here we present the spectral functions for various $\mathrm{k}$-vectors in a generic simple

Iig. 2 P.M-derived spectral functions at the indicated points in the simple cubic Brillouin zone, for two temperatures relative to $T_{k}$. Dark lines indicate partial $f$-DOS while gray lines indicate partial d-DOS. Note that the narrowest features, both $f$ and $d$, are at the Fermi energy. 
cubic rone along the cube diagonal, at two different temperatures $\left(0.46 \mathrm{~T}_{\mathrm{K}}\right.$ and $\left.3 \mathrm{~T}_{\mathrm{K}}\right)$. $\mathrm{A} \mathrm{d}$ band at 0.6 filling is allowed to hybridize with a band of singly occupied f-states, resulting in the two $[-d$ mixed renormalized bands, with a clear hybridization gap. For this system, $U=$ 1.5 , hybridization $\mathrm{V}=0.6$, while $\mathrm{TK}=0.02$, in units of $\mathrm{t}^{*}$. Clearly dispersion is much larger than $T_{k}$. There is no $f$-intensity at $E_{F}$ for $k=(0,0,0)$, the latter developing only at $k=$ $(1,1,1) \mathrm{p} / 2$. As the temperature increases to $3 \mathrm{~T}_{\mathrm{K}}$ there is only a minimal transfer of spectral weight from the quasiparticle peaks to the "main" or $f^{0}$ peak (still slightly visible at $w \approx-1$ ) in contrast to the SIM. Instead one finds primarily a broadening of the quasiparticle peaks, very much in accordance with experimental results reported previously [see papers by Arko, Joyce and Andrews]. Calculations for $T=10 T_{K}$ yield similar results. As in the SIM, the $f^{0}$ peak, despite much reduced intensity, is found to increase with decreasing hybridization, and hence $\mathrm{T}_{\mathrm{K}}$. The single impurity physics is generally recovered at sufficiently high temperatures ( $\mathrm{T}>$ $\left.10 \mathrm{~T}_{K}\right)$.

\section{EXPERIMENTAL RESULTS}

\section{Single Crystal vs Poly Crystal PES}

As indicated in the introduction, the emphasis in this chapter will be primarily on single crystal photoemission spectra and their comparison to the various models. It may be useful, however, to show from the start that indeed major differences exist between single crystal and poly-crystal heavy fermion PES spectra, in order to demonstrate the need for a new approach.

In Fig. 3 we show single- and poly-crystal spectra for $\mathrm{YbCu}_{2} \mathrm{Si}_{2}$ where the $\mathrm{KR}$ is identified with the $4 \mathrm{f}_{7 / 2}$ state and is fully occupied [Arko et. al., unpublished]. The samples. were cleaved (not scraped) in-situ at $20 \mathrm{~K}$, and measured at $20 \mathrm{~K}$. The $\mathrm{T}_{\mathrm{K}}$ for this material is approximately $35 \mathrm{~K}$. The spectra have been normalized so that the trivalent portion of the spectrum (presumed to be entirely due to the bulk) has equal intensities in both curves when measured peak to valley. While the $\mathrm{Cu}-3 \mathrm{~d}$ feature at $\mathrm{hv}=-4 \mathrm{eV}$ normalizes in this way to equal intensity in both materials (the slight shifts are due to angle resolved effects), the divalent portion of the $\mathrm{Yb}-4 \mathrm{f}$ spectrum within $2 \mathrm{eV}$ of the Fermi energy is dramatically reduced in the poly-crystalline specimen relative to the trivalent portion, both for surface and bulk features. In terms of the $4 \mathrm{f}$ hole occupancy discussed below, the $\mathbf{n}_{\mathrm{f}}$ is apparently increased from 0.65 to about 0.8 or 0.9 in the poly-crystal. Stoichiometry does not seem to be the problem since the $\mathrm{Cu}-3 \mathrm{~d}$ peak has the correct intensity. If the SIM truly applied in $\mathrm{Yb}$ heavy fermions, then all the $4 \mathrm{f}_{7 / 2}$ intensity at $\mathrm{E}_{\mathrm{F}}$ is of necessity due to the Kondo interaction. The SIM by definition implies that the $4 \mathrm{f}$ element is an impurity with no interaction with its neighbors at high temperatures. Why the existence of long range atomic order (or lack thereof) then so dramatically affects the $4 \mathrm{f}$ spectrum is at this point a mystery. Some suggestions will be presented later in the section 13 where we consider the possibility that these are band states cren at high temperatures, and long range order may be impaired in poor poly-crystals.

It is natural to suspect poor sample quality in poly-crystals for the above result, and this is certainly expected to be a contribution. However, although the quenching of the $4 \mathrm{f}_{5 / 2}$ leature in poly-crystals is not totally universal, it occurs of ten enough that an effect other than poor sample stoichiometry is a contributing factor. On the other hand, inasmuch as it is single crystal data that is more out of line with bulk properties than poly-crystal data, it is occasionally suggested that this could be due to a surface effect in single crystals. Unfortunately this runs counter to conventional wisdom which states that best coupling to the bulk is obtained in single crystals at normal emission. Indeed, poly-crystals, especially scraped poly-crystals, are far more likely to exhibit strong surface effects. Further, only the KR and its sidebands are affected by the poly-crystal and scraping, the trivalent portions of the spectra remaining unaffected. Clearly we are hinting that the $4 f$ states exist in very narrow bands whose phase coherence is readily destroyed by an imperfect surface.

To sum up, it is apparently crucial for a correct measurement of the near- $E_{\mathrm{F}}$ intensities in heavy fermions that the surface is a cleaved single crystal, with a mirror surface especially desirable. Scraping of the surface should be particularly avoided since it was found that in $\mathrm{YbAgCu}_{4}$, a matcrial having a $\mathrm{T}_{\mathrm{K}}$ of about $35 \mathrm{~K}$, the scraping of a coarsc-graincd surface totally climinated all divalent $4 f^{\circ}$ features [Arko et. al., unpublished], much more consistent 
Fig. 3 Single crystal and poly-crystal $\mathrm{YbCu}_{2} \mathrm{Si}_{2}$ PESspectra taken under identical conditions Note the large reduction in poly-crystal divalent intensity even though the trivalent intensities are equal.

with thermodynamic data. Perhaps it is so that the destruction of long range order via scraping indeed puts us into the single impurity regime, while a good single crystal surface with long range order requires a treatment within the PAM.

\section{Lack of Scaling with $T_{K}$ of the Near- $E_{F}$ Features}

Perhaps the single most important prediction of the GS and NCA models is that of: scaling of the various spectral properties with hybridization and hence $T_{K}$, the universal parameter. In particular, the spectral weight, position, and width of the $K R$ is predicted to increase with $T_{K}$. While instrument resolution has been insufficient in the past to deal properly with the position and width of the $4 f_{5: 2}$ feature, researchers have concentrated on the spectral weight. Allen et. al. [1986] were first to find a relationship between $T_{K}$ and spectral weight of the near- $E_{\mathrm{F}}$ features, although the limited resolution of the early research did not allow a quantitative comparison to theory.

Patthey et. al. [1990] published a monumental paper on the application of the SIM to spectral properties of $\mathrm{Ce}$ and $\mathrm{Yb}$ heavy fermions. For the first time they performed high resolution ( $\Delta E \approx 20 \mathrm{meV}$ ) systematic experiments on a number of materials with $T_{K}$ 's ranging from less than $10 \mathrm{~K}$ for $\mathrm{CeCu}_{6}$ to about $200 \mathrm{~K}$ for $\alpha$-Ce, observing the trends in the near$E_{F}$ spectral weight. They obtained their data using HeI and HeII radiation, using the difference curves obtained by subtracting the spectra obtained at two different photon energies. More importantly, they also performed model calculations using NCA at the appropriate temperatures to compare to the results and found excellent agreement.

Joyce et. al. [1992c], by contrast, pointed out that by using high quality single crystals there obtains an amazing similarity between all Ce hear y fermion spectra, regardless of $T_{k}$. This can be seen in Fig. 4 [Arko et. al., 1997] where spectra for 7 Ce heavy fermions (including $1 \%$ ) directions for $\mathrm{CeBe}_{13}$ ) are plotted. Indeed, one observes the interesting effect that spectral differences with momentum within the same crystal $\left(\mathrm{CeBe}_{13}\right)$ are in fact larger than differences between materials whose $T_{K}$ 's differ by as much as 2 orders of magnitude (more on that later). All the spectra in Fig. 4 were taken at a photon energy of $120 \mathrm{eV}$ which roughly corresponds to the $4 \mathrm{~d}$ absorption edge and thus resonantly enhances the $4 \mathrm{f}$ emission. The encrgy resolution in cach case is about $90 \mathrm{mcV}$. All spectra represent raw data cxcept in the casc of $\mathrm{CcCu}_{2} \mathrm{Si}_{2}$ where the spectrum at anti-resonance $(\mathrm{hv}=112 \mathrm{eV})$ has been subtracted from the resonance spectrum in order to eliminate the very strong $\mathrm{Cu}-3 \mathrm{~d}$ emission. In Fig. 4 one should further note that $\mathrm{CeAl}_{3}$ and $\mathrm{CeSi}_{2}$ are cleaved poly-crystal specimens so that the poly-crystal effect discussed above is not universal (but common). The normalization of the spectra in Fig.4 is to the $f^{\circ}$ peak maxima at -2 to $-3 \mathrm{eV}$, except for $\mathrm{CeBe}_{13}(110)$ where the $\mathrm{f}^{0}$ pcak is clcarly much smaller than that for $\mathrm{CeBe}_{13}(100)$, and it just is not obvious which maximum is the true bulk $\mathrm{f}^{\circ}$ (this is further discussed below). While nearly all the spectra in Fig. 4 represent $4 \mathrm{f}$ and $5 \mathrm{~d} \mathrm{Ce}$ emission only (due to the nearly negligible ligand emission), in the case of $\mathrm{CePt}_{2+x}(111)$ and $\mathrm{Ce}_{3} \mathrm{Pt}_{4} \mathrm{Sb}_{4}$ there is also substantial Pt-5d emission so that the $\mathrm{I}^{0}$ peak intensities should be ignored for these. To the list of spectra in Fig. 4 one should also 
Fig. + PES spectra for several $C e$ heavy fermions with $T_{K}$ 's ranging fron $\approx 1 \mathrm{~K}$ to $\approx 400 \mathrm{~K}$. Note the lack of scaling of the $\mathcal{H}_{5,2}$ with $\mathrm{T}_{K}$. Two different directions are shown for $\mathrm{CeBe}_{13}$.

Fig. 5 Overlay of $\mathrm{CeBe}_{13}(110)$ spectrum with $\mathrm{CePt}_{2+\mathrm{x}}(001)$ spectrum after subtracting backgrounds (from Andrews et al. [1995a]. The calculated GS spectrum approximates the parameters for $\mathrm{CeBe}_{13}$.

add $\alpha$-Ce $\left(\mathrm{T}_{\mathrm{K}} \approx 800 \mathrm{~K}\right.$, Weschke et. al., [1991]) and $\mathrm{CeB}_{6}\left(\mathrm{~T}_{\mathrm{K}} \approx 10 \mathrm{~K}\right.$, Kakizaki, [1995]) which likewise exhibit spectra nearly identical to those in Fig.4.

Irrespective of the precise normalization of the data in Fig. 4, the interesting fact cmerges that the spectral weights of the $4 \mathrm{f}_{5 / 2}$ features are more or less constant for all materials, or at least do not monotonically increase with $T_{K}$. While the $90 \mathrm{meV}$ resolution precludes any firm conclusions about the widths and positions of the $4 f_{s / 2}$, it has little effect on the broad $4 \mathrm{f}_{72}$ peaks so that their peak amplitudes relative to $\mathrm{f}^{0}$ need no deconvolution. One is forced to the conclusion that there is no monotonic scaling of the $4 f_{5 i 2}$ spectral weights with $T_{K}$ relative to the $f^{0}$ peak. This lack of scaling cannot be blamed, as has been discussed above [Joyce and Arko, 1993a], on inclusion of $\mathrm{f}^{2}$ intensity originating from finite $U_{\mathrm{ff}}$ effects, since the $\mathrm{f}^{2}$ DOS, if present, is broad and featureless and hence cannot change the relative amplitudes. Nor can one resort to a lowering of the $4 f_{5 / 2}$ degeneracy to $N_{f}=2$ by the introduction of two additional crystal field levels, since, as again shown by Joyce and Arko [1993a], even in the presence of these CF states the GS and NCA models predict a scaling of the $4 \mathrm{f}_{5 / 2}$ spectral weight with $T_{\mathrm{K}}$. The nearly constant and much too broad (relative to GS) width of the $4 f_{52}$ has becn attributed by Patthey et. al. [1993] to lifetime broadening. Even if this were the case, such broadening will not affect the spectral weight so that the inevitable conclusion of a lack of scaling with $T_{K}$ remains a reality.

To. underseore the lack of scaling still further we point out the amazing similarity in Fig. 4 between $\mathrm{CeBe}_{13}(110)\left\{\mathrm{T}_{\mathrm{K}} \approx 400 \mathrm{~K}\right\}$ and the spectrum for $\mathrm{CePt}_{2+\mathrm{x}}(111)\left\{\mathrm{T}_{\mathrm{K}} \approx 10 \mathrm{~K}\right\}$. In Fig. 20) the comparison is somewhat less striking than actuality because of the strong Pt$5 \mathrm{~d}$ cmission in the region of the $f^{\circ}$ peak. For a better comparison one must first subtract out the non-Ce portion of the CePt (111) spectrum by subtracting the hv $=112 \mathrm{eV}$ spectrum (anti-resonance) from the $h v=120 \mathrm{eV}$ spectrum (resonance). For $\mathrm{CeBe}_{13}$ the intensity at 112 $\mathrm{cV}$ is negligible. The two curves are overlaid in Fig. 5 (normalized at the $4 \mathrm{f}_{5 ; 2}$ peak), with the sccondary backgrounds also removed. With the exception of the shift in the $\Gamma^{\circ}$ peak the two curves overlay almost exactly. The region of the Ce-5d emission (between $-0.5 \mathrm{eV}$ and -2 $\mathrm{eV}$ ) also overlays if the $\mathrm{f}^{0}$ peaks are removed. This type of agreement is astounding when one considers that there is a factor of 40 difference in $\mathrm{T}_{\mathrm{K}}$ 's. Moreover, the $\mathrm{CePt}_{2+x}(111)$ spectrum was laken at $120 \mathrm{~K}$ where the KR should have been of negligible intensity. On the basis of the single crystal data, then, it would be difficult to reach a conclusion other than one of a total lack of scaling of the spectral weight with $T_{K}$.

The thin lined spectrum in Fig.5 is a GS calculation with parameters approximately matching $\mathrm{CeBe}_{13}$. (i.e., $W=10 \mathrm{eV}, \mathrm{U}_{\mathrm{ff}}=14 \mathrm{eV}, \mathrm{T}_{\mathrm{K}}=400 \mathrm{~K}$ ), smoothed to match the instru- 
ment resolution, and normalized in such a way that the total area of the calculated Lorentian $f^{(1)}$ peak matches the total arca of the measured Gaussian $f^{0}$ peak in $\mathrm{CeBc}_{13}$. The mismateh is obrious. While better tits can be obtained with different parameters such as a smaller $W$. these are diflicult to justify. The width of the conduction band used above (10 eV) may in fact be cien conservative since experimentally it peaks at about $-8 \mathrm{eV}$. Also, there are no $\mathrm{Cc}$ nearest neighbors in $\mathrm{CeBe}_{13}$ so that the 4 f electron can only hybridize with the $\mathrm{Be}$ conduction band.

\section{The f-level Occupancy, $n_{r}$}

It can alrcady be scen in Fig. 5 that the spectral weight ratio $\mathrm{W}_{\mathrm{r}}=\mathrm{f}^{\mathrm{t}} / \mathrm{f}^{0}$ in $\mathrm{CcBc}_{13}$ is too large relative to a calculation; indeed, about a factor of 2.5 too large. The discrepancy must then be cren larger for $\mathrm{CePt}_{2.2}$. This indicates that the calculated f-occupancy $\mathrm{n}_{\mathrm{f}}$ of the bare $\mathrm{f}$ level at $\approx-2.5 \mathrm{cV}$ is much larger than measured. One can, of course vary the parameters (primarily increase the hybridization) until calculation matches experiment, but this will increase $T_{K}$ to values incommensurate with the thermodynamic quantities. Obtaining a good fit for onc compound by varying parameters says very little about the necessary systematics. Basically, the lack of scaling seen in Fig.5 implies that the $n_{\mathrm{f}}$ in Ce compounds does not change very dramatically with $\mathrm{T}_{\mathrm{K}}$. This is totally at odds with the SIM expectations.

The f-level occupancy is best studied in $\mathrm{Yb}$ compounds where it becomes the hole occupancy rather than electron occupancy, and where GS theory places the KR centroid on the occupicd side of the Fermi encrgy. Here the integrated intensitics of the $\mathrm{f}^{13}$ (trivalent) and $\mathrm{f}^{1+}$ (divalent) portions of the photoemission spectrum are expected to be directly related to the $f$ hole occupancy, $n_{f}$, by the relation

$$
\mathrm{n}_{\mathrm{f}}=\mathrm{I}\left(\mathrm{f}^{13}\right) /\left[\mathrm{I}\left(\mathrm{f}^{13}\right)+13 / 14\left(\mathrm{f}^{14}\right)\right]
$$

where $I\left(f^{n}\right)$ represents an integral spectral weight of the $f^{\mathrm{n}}$ feature. One of the best compounds for this study is $\mathrm{YbAl}_{3}$ where the $\mathrm{Al}$ states yield almost no photocurrent, thus making: : it relativcly casy to subtract the background and to delincate the trivalent and divalent (bulk) intensities. An early study for this material [Oh, 1993] was an XPS study where direct integration yiclded $n_{\mathrm{f}}=0.65$ which is far lower than cxpected for a material having $T_{\mathrm{K}} \approx 400 \mathrm{~K}$. Indeed this places it well into the mixed valent regime, outside the range of the validity of NCA. Tjeng et. al. [1993] later utilized the temperature dependence of the $4 f_{7 / 2}$ level and obtaincd an $n_{f}$ valuc of 0.85 which is in exact agrecment with NCA predictions [Bickers et. al., 1987]. However, using the temperature dependence is difficult at best. The assumption is made that all of the temperature dependence of the $4 f_{7 / 2}$ is due to Kondo effect. With no effort made to delincate the cffects of a $300 \mathrm{~K}$ Fermi function combined with thermal broadening, this assumption seems tenuous at best. Additionally the choice of backgrounds in that study is somewhat questionable. Since the non- $4 f_{7 / 2}$ (bulk) background in the polycrystalline samples was assumed to comprise more than $80 \%$ of the signal, one can sec how even in the case of correct assumptions regarding temperature dependence, small errors in the choice of background can yield huge errors in the temperature dependence.

The most reliable method for determining $n_{\mathrm{f}}$ would seem to be direct integration of the divalent and trivalent features in a material where the background is relatively small. Toward

Fig. $6 \mathrm{XPS}$ and $\mathrm{PSS}(\mathrm{hv}=120 \mathrm{eV})$ spectra for $\mathrm{YbAl}_{3}$ taken at $\mathrm{T}=20 \mathrm{~K}$ to determine any differences in signal due to escape depth. Nearly identical values of $\mathbf{n}_{f}$ were obtained by direct integration after separating out the background and surface features as shown 
this cnd Joyce et. al. [1996] and Andrews et. al. [1994] analyzed YbAl, spectra both from XPS as wcll as UPS and came up with values basically agreeing with the first investigation of Oh [1993]. This is shown in Fig. 6 where the delineation into background and surface components is also illustrated. Similar analyses for $\mathrm{YbCu}_{2} \mathrm{Si}_{2}$ yielded [Andrew's et. al., 1994 and Joyce et. al., 1996] nearly the identical values despite the fact that $\mathrm{YbCu}_{2} \mathrm{Si}_{2}$ has a $\mathrm{T}_{\mathrm{K}}$ of only $35 \mathrm{~K}$. an order of magnitude lower than $\mathrm{YbAl}_{3}$. All of these values are totally out of the range of $\mathrm{VCA}$ ralidity and clearly belong to the mixed valence regime. While there remains some question as to whether the photoelectron cross sections for the $\mathrm{f}^{13}$ and $\mathrm{f}^{1+}$ states are identical (a necessary condition in order to compare intensities), the fact that identical $n_{f}$ values are obtaincd at both XPS cncrgics as well as ncar $h v=100 \mathrm{cV}$ would suggest that the photoclectron cross sections of the $\mathrm{f}^{13}$ and $\mathrm{f}^{1+}$ states are at least very similar if not identical. Thus direct integration of the spectra to obtain $n_{\mathrm{f}}$ would seem preferable to the method of Tjeng ct. al. [1993].

PAM calculations indicate that the ratio of the $\mathrm{f}^{1}$ to $\mathrm{f}^{0}$ (or, the divalent to trivalent in Yb compounds) spectral weights is substantially larger when calculated within the PAM than when calculated within the SIM. It would secm that the trends predicted by the PAM are much more consistent with the experimental spectra obtained from single crystals.

There is no question about the fact that the $n_{\mathrm{f}}$ values obtained from PES are dramatically in disagrecment with those obtained from bulk propertics as well as those obtaincd from $\mathrm{X}$-ray absorption $\mathrm{L}_{\mathrm{m}}$ edge studies. While it is our contention that we must resort to a different model, such as the PAM, to resolve this discrepancy, a useful question to ask is whether photocmission mcasures the same material as is measured in $\mathrm{L}_{\mathrm{m}}$ studies. In particular, is it possible that owing to its surface sensitivity a PES study does not probe the bulk but rather only a surface or a sub-surface layer whose $n_{f}$ is dramatically different from the bulk. Such a scenario would render PES uscless and would breathe new life into SIM.

But let us look at all possibilities. The existence of a surface layer is not in question. Moreover it is clear that in Ce compounds the surface tends to be trivalent while in $\mathrm{Yb}$ compounds it is divalent. But while it is casy to delincate a surface feature and scparate it from bulk electronic structure, a thick subsurface has never yet been reported in any system known to us (thin subsurfaces may be preset in $\mathrm{Yb}$ compounds as evidenced by multiple surface features). If it were to cxist in one system, the likelihood of its existence in other systems is high, making PES useless as a bulk probe. Fortunately, excellent agreement between calculated and PES-derived electronic structure is of ten reported. Indeed, most calculations suggest that only about 3 atomic layers below the surface are sufficient to obtain the bulk electronic structure.

But let us say for the sake of argument that a sub-surface layer may exist only in strongly corrclated f-clcctron systems. Immediately we can rule it out in $\mathrm{Cc}$ compounds since studies at photon energies as high as $3 \mathrm{KeV}$ show only bulk and surface states with no third layer. Such a sandwich would in any case be preposterous for Ce heavy fermion compounds since it would require a trivalent surfee, a strongly mixed valent sub-surface, and again a nearly trivalent bulk. We can also rule it out in purely trivalent as well as purely divalent $\mathrm{Yb}$ compounds since PES measurements yield the required purely trivalent $\left(\mathrm{YbBe}_{13}\right)$ or purely divalent $\left(\mathrm{YbSn}_{3}\right)$ spectra. It would be pathological that a sub-surface would exist only in mixed $\backslash a$ lent $Y b$ compounds, but that is all that we are left with. Even here we see from the previous section that scraping of a surface only affect the divalent portion of the spectrum i.c., the wherent bands, with little effect on the trivalen spectrum.

But let us go further and assume that nature is indeed that pathological. Since the $n_{f}$ values obtaincd at XPS energies are the same as those obtained at lower photon energies, this suggests that the desired sub-surface must be at least of the order $100 \AA$ thick otherwise the larger escape depth at XPS energies would begin to probe the supposed true bulk and yield different $n_{f}$ yalues. On the other hand it must not be so thick as to constitute a significant percentage of the probing depth in $\mathrm{L}_{\mathrm{m}}$ edge studies where-a sub-surface has never been observed (again pathological). But the real question to be asked is the following: If a single crystal compound is cleaved in-situ at about $20 \mathrm{~K}$, what is the driving force which causes this $\approx 100 \AA$ thick layer to form instantly, and why is the LEED pattern representative of the bulk structure? Certainly there are no dangling bonds as on the surface. The answer remains elusive.

One is forced to the conclusion that there exists no sub-surface and that the measured $n_{f}$ values are real. The disagreement with $L_{m}$ studies remains unresolved. Perhaps we should 
look to problems associated with $\mathrm{L}_{\mathrm{in}}$ edge measurements rather than PES measurements, particularly in sicw of the problems uncovered recently with bandwidths.

\section{Temperature Dependence}

General Considerations: The determination of the temperature dependence of the

ncar- $E_{F}$ fature in heavy fermion compounds is perhaps the most difficult experiment to accomplish correctly. Eicry matcrial (cven Cu, [Matzdorf, 1993]) displays a temperature dependence to some degree in its electronic structure, and it is difficult to determine whether the behavior observed is due to conventional effects of lattice contraction and phonon broadening, or whether it is due to the unconventional behavior predicted by the NCA.

The difficulty of the experiment has precluded much investigation in this arena outside the single crystal work of Joyce et. al. Indeed, in poly-crystalline materials the only PES studies are on $\mathrm{CcSi}_{2}$ [Patthcy ct. al., 1987], $\mathrm{YbAl}_{3}$ [Tjeng ct. al., 1993] and $\mathrm{YbAgCu}_{4}$ [Weibel et. al., 1993], while some BIS data exist for $\mathrm{CeSi}_{2}$ [Malterre et. al., 1992a; Grioni et. al, 1993] and $\mathrm{CePd}_{3}$ [Malterre, et. al., 1992b]. In each of the above studies it was determined that the temperature dependence of the ncar- $\mathrm{E}_{\mathrm{F}}$ feature follow's the single impurity model exactly. However, they all have in common the fact that the surfaces are those of scraped poly-crystals. Moreover, there is the ever present problem of the choice of a background which can be varicd by more than a factor of two thus leading to nearly any desired result. In none of the experiments above was the background determined from measurements of related La or Lu compounds to properly delineate first the non-f contribution, and second, the contribution duc to the ever present surface components. In general, the background is grossly over estimated with the assumption that all the spectral intensity at the valley immediatcly bclow the ncar- $E_{\mathrm{F}}$ peak (i.c., all spectral weight at $\approx-0.2 \mathrm{cV}$ in Fig. 23) is duc to background. By drawing a horizontal line between this valley and the Fermi energy and calling it: background perhaps half of the $4 f_{7 / 2}$ peak intensity is eliminated. Clearly this will exaggerate any temperature dependence.

Fig. $7 \mathrm{VCA}$ calculations at three different temperatures for three materials discussed here. Surface states are not modeled by $: \mathrm{VA}$. The parameter used are: $\left(\mathrm{Yb} \mathrm{AgCu}_{4}\right) \mathrm{T}_{\mathrm{K}}=100 \mathrm{~K}, \mathrm{U}_{\mathrm{ff}}=\infty, \mathrm{W}=6.0 \mathrm{cV}, \mathrm{D}_{\mathrm{so}}=1.29 \mathrm{cV}$, $\mathrm{e}_{\mathrm{f}}=1.0 \mathrm{eV}, \mathrm{CF}$ staes - none, $\mathrm{G}=0.05+62 \mathrm{eV} .\left(\mathrm{YbCu}_{2} \mathrm{Si}_{2}\right) \mathrm{TK}=40 \mathrm{eV}, \mathrm{U}_{\mathrm{ff}}=\infty, \mathrm{W}=6.0 \mathrm{eV}, \mathrm{D}_{\mathrm{sO}}=1.29$ $\mathrm{eV}, \mathrm{e}_{\mathrm{f}}=1.0 \mathrm{cV}, \mathrm{D}_{\mathrm{CF} 1}=12 \mathrm{meV}, \mathrm{D}_{\mathrm{CF}}=30 \mathrm{meV}, \mathrm{D}_{\mathrm{CF}}=80 \mathrm{meV}, \mathrm{G}=0.0606 \mathrm{eV} .\left(\mathrm{YbAl}_{3}\right) \mathrm{T}_{\mathrm{K}}=400 \mathrm{~K}, \mathrm{~L}_{\mathrm{ff}}$ $=x . \mathrm{HF}=10.0 \mathrm{eV}, \mathrm{D}_{\mathrm{so}}=1.29 \mathrm{eV}, \mathrm{e}_{\mathrm{i}}=1.0 \mathrm{eV}, \mathrm{CF}$ staes - none, $\mathrm{G}=0.05856 \mathrm{eV}$.

Fig. 8 Experimentally measured temperature dependence between $20 \mathrm{~K}$ and $80 \mathrm{~K}$ for the three materials calculated in lig. 7. 
Yb Compounds - Comparison to NCA: The NCA predictions (code provided by D. Cox) for the temperature behavior of these materials are show $n$ in Fig. 7 [from Josce ct. al., 1996]. Here the $4 f_{72}$ and $4 f_{5,2}$ features are shown without the omni-present surface states which cannot be modeled by NCA. The three temperatures for which calculations exist are those most casily accessible experimentally. The parameters are given in the figure caption, but are chosen so as to approximate the three matcrials and their respective $T_{K}$ 's. The data has also becn smoothed with a $190 \mathrm{mcV}$ Gaussian to match the resolution of the $\mathrm{YbCu}_{2} \mathrm{Si}_{2}$ data shown in the bottom frame of Fig. 8. In all cases a large temperature dependence is predicted. In particular, note the large decrease in intensity between $20 \mathrm{~K}$ and $80 \mathrm{~K}$ for $\mathrm{YbAgCu}_{4}$, a matcrial having a $\mathrm{T}_{\mathrm{K}}$ in the vicinity of $100 \mathrm{~K}$.

By contrast, Fig. 8 shows that the measured temperature dependence between $20 \mathrm{~K}$ and $80 \mathrm{~K}$ in all three materials [Blyth et. al., 1993] is almost nil, with the surface states usually showing larger cffects than the bulk states. Here the $\mathrm{YbAgCu}_{4}$ specimen was a coarse graincd poly crystal clcaved in-situ. The results contrast sharply with the measurements of Malterre et. al. [1992a] who reported a large temperature effect in $\mathrm{YbAgCu}_{4}$ between $20 \mathrm{~K}$ and $80 \mathrm{~K}$. A gain, however, in view of the superionty of single crystal data (or at lcast clcaved vs scraped surfaces) more wcight is given to results from clcaved surfaces, the scraped polycrystal data having too many uncertainties. Indeed, Arko et. al. [unpublished] observed that scraping their $\mathrm{YbAgCu}_{4}$ surfacc totally destroys the $4 \mathrm{f}_{7 / 2}$ and $4 \mathrm{f}_{5 / 2}$ bulk pcaks which were present on the same specimen when the surface was preparcd by cleaving. The intensitics slowly recover with time and a temperature dependence can be observed below $80 \mathrm{~K}$, but the surfacc composition preparcd in this fashion surcly is no longer representative of the starting material:

Above $80 \mathrm{~K}$ a substantial temperature dependence is usually obscrved even in single crystal $\mathrm{Yb}$ heavy fermions, albeit not always. Spectral weight changes ranging from $0 \%$ to about $15 \%$ are common. Joyce et. al. [1992c] suggested already in connection with $\mathrm{CeSi}_{2}$ data of Patthey ct. al. [1987] that onc should consider thermal broadening as a source of tempcrature dependencc. In reply it was pointed out [Patthey ct. al., 1993] that while core level phonon broadening can be understood within the Franck-Condon model, the near- $E_{F}$ features in heavy fermions should not couple to the phonons owing to the well-screcned condition of these states. Nevertheless, phonon broadening is observed in the valence bands of many materials, even in excellent metals like $\mathrm{Cu}$ [Matzdorf et. al., 1993], where the screening is expected to be good. Thus, while not entirely understood, phonon broadening is an existing fact and must be considered. In $\mathrm{CeSi}_{2}$ Joyce et. al. [1992c] estimated the magnitude of the broadcning (about $100 \mathrm{mcV}$ ) from the broadcning of the Si-2p corc levels. Whilc this is certainly not expected to be identical to the Ce states, it should serve as a first order approximation. In $\mathrm{Yb}$ compounds the phonon broadening was estimated from a measurement of pure $\mathrm{Yb}$ metal, and found to be of the order of $130 \mathrm{meV}$ added in quadrature.

Fig. 9 Simulated temperature dependence of a fitted $\mathrm{YbAl}_{3}$ spectrum A $12.5 \%$ spectral

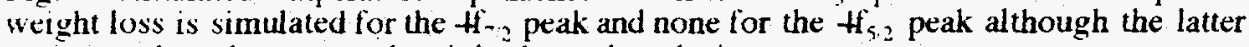
appears to have lost spectral weight due to broadening. 
Assuming that a similar $130 \mathrm{meV}$ broadening occurs in heavy lermion compounds where the $4 f_{7_{2}}$ is within 20 to $30 \mathrm{mcV}$ of the Fermi cnergy, one can then simulate the effect of the Fermi lunction on the $4 f_{72}$ intensity [Joyce et. al., 1996]. This is done in Fig. 9 where fits to the bulk divalent $4 f$ peaks of Fig. 28 (i.e., the bulk $4 f_{7}$ and $4 f_{52}$ ) measured at $20 \mathrm{~K}$ are broadencd by $130 \mathrm{meV}$ in quadrature and convoluted with a $300 \mathrm{~K}$ Fermi function. The middle frame hows the effect near the Fermi energy using a $45 \mathrm{meV}$ experimental resolution, while the buttom trame gives the same result with $100 \mathrm{meV}$ experimental resolution. The calculated spectral weight loss of the $\mathrm{Yb}-4 \mathrm{f}_{72}$ peak is about $15 \%$ which is actually larger than the observed result. Thus the broadening in compounds is perhaps somewhat less than 130 $\mathrm{mcV}$, indecd most likcly closer to $100 \mathrm{mcV}$ as found [Joyce ct. al., 1992c] in $\mathrm{CcSi}_{2}$. It is important to note from the bottom frame of Fig. 9 that given some degree of data scatter one could never unambiguously distinguish between peak broadening and spectral weight loss, although a carcful subtraction of the backgrounds of ten yiclds a zcro weight loss for the $\mathrm{Yb}$ $4 \mathrm{f}_{52}$ even in the presence of a $10 \%$ weight loss for the $\mathrm{Yb}-4 f_{7 / 2}$. In Fig. 27 no data analysis is needed to see this effect.

Thermal broadening and Fermi function effects work well for Cc compounds as well, but space does not permit a detailed examination.

\section{YbInCu, A Singular Test of the Single Impurity Model}

Y bInCu4 provides the best opportunity to date for testing the predictions of the SIM. Not only is it an $\mathrm{Yb}$ compound where the Kondo features (in the Kondo limit) are on the occupied side of the Fermi energy, but also it undergoes an isostructural phase transition at $T_{V}=42 \mathrm{~K}$ with a $0.5 \%$ volume reduction below this temperature. It was initially suspected that this is the $\mathrm{Yb}$ analogue of the Ce Kondo volume collapse, but it quickly became apparent (Lawrence et al. ?) that subtle changes in the density of states were responsible for the phase transition. Nonetheless, within the SIM framework the Kondo temperature changes at the transition from a value of $\approx 25 \mathrm{~K}$ above $T_{v}$, to a value of $\approx 400 \mathrm{~K}$ below $T_{v}$. This more than an order of magnitude change in $T_{K}$ allows for easy testing of the NCA predictions within the same material. In particular one can measue: $n_{f}$, temperature dependence of spectral weight, linewidths and their changes predicted by NCA, peak position shifts, etc. In particular, $\mathrm{n}_{\mathrm{f}}$ is expected to change from a value of about 0.8 below $T_{v}$ to a value $>0.9$ above $T_{v}$, the intrinsic linewidth should narrow by an order of magnitude above $T_{v}$, the peak position of the spinorbit peak (as well as the $\mathrm{K}_{\mathrm{R}}$ whose exact position is complicated by the Fermi function) should shift toward $E_{F}$ by $40 \mathrm{meV}$ above $T_{V}$.

In Fig. 10 are shown a series of spectra at $45 \mathrm{meV}$ resolution for temperatures ranging from $20 \mathrm{~K}$ to $200 \mathrm{~K}$, thus covering adequately the $T_{V}$ region. Even a cursory visual inspection reveals that there cannot possibly be an order of magnitude change in the spectral weight of the bulk features above and below $T_{\vee}$. Joyce et al. (1988) have carefully measured the $n_{f}$ at $80 \mathrm{~K}$ (above $T_{v}$ ) and found a value of 0.5 , far below the expected value of $>0.9$ cxpected from NCA. Below $T_{V}$ this value decreases to about 0.4 . Immediately, too, one can see that there is no shift at all in the SO peak position.

It is periodically suggested that that the surface sensitivity of PES measurements woukd render these results useless if a thick sub-surface layer prevents electron escape from the true bulk into the vacuum. In that instance we would be seeing perhaps a thick subsurface w hose $T_{K}$ is much larger than the bulk and where no transition occurs at $T_{V}$. We have already hinted that such a thick sub-surface layer would have to be pathological to $\mathrm{Yb}$ compounds. Further there is absolutely no evidence for its existence except for the disagreement with SIM. A thin (one atomic layer) sub-surface in $\mathrm{Yb}$ compounds is a possibility owing to the obscrvation of several surface states, but in all cases the bulk is clearly visible. We suggest that it is not the PES measurement that is in error, but rather the SIM and possibly $\mathrm{L}_{\text {III }}$ cdge studies..

It is easy to err when analyzing PES data. In order to measure the spectral weight of the near- $E_{F}$ leatures, a straight line background (used, e.g., by Tjeng et al., 1993) is of ten cmployed to climinate the non-f portion of the spectral weight. This is shown as the dotted line in Fig. 10. But the real background can only be obtained by measuring a corresponding Lu compound under identical conditions. In that case the non-bulk, non-4f portion of the spectrum corresponds to the thick gray curve in the $20 \mathrm{~K}$ spectrum of Fig. 10. Thus the "Tjeng" background arbitrarily eliminates as much as $50 \%$ of the true bulk spectral weight of 
Fig. 10 Normal emission ARPES spectra for $\mathrm{YbInCu} 4$ at indicated temperatures. The SO intensity is Tindependent above $50 \mathrm{~K}$. Gray curve in $20 \mathrm{~K}$ spectrum accounts for surface features and non-f states, and is thus the best estimate of the background. Straight line background is shown for comparison

Fig. 11 Comparison of $4 f_{5: 2}$ and $4 f_{-2}$ intensities analyzed by two methods. Integrated intensities are $T_{3}$ : independent above the bulk transition. Peak heights are measured from the straight line background.

the $4 f_{7 / 2}$ (shaded area) and leads to an erroneously enhanced temperature dependence. The crror is compounded when no curve fitting cmployed to get the integrated spectral weight. Instead, only peak heights are measured from this background.

Even emplying curve fitting and a correct background, to get the true $4 f_{7 / 2}$ spectral weight requircs taking into account the effect of the Fermi function and theremal broadening. Eicry researcher, but we, has ignored this non-trivial effect. For this reason we suggest that it is actually easier to measure the weight of the $4 f_{5 / 2}$ peak where thermal broadening effects are lcss significant, and there is no Fermi function to dcal with. Indecd, simple peak height analysis is almost sufficient as shown in Fig. 11. Here we present intensity data at various temperatures for both the $4 \mathrm{f}_{722}$ and the SO peaks, analyzed both from simple peak heights (using the Tjeng background) as well as by integrating fitted peaks. Clearly a peak height analysis using a Tjeng background overestimates the temperature dependence of the $4 f_{72}$ by about $120 \%$. Even the integrated intensity analysis for the $4 \mathrm{f}_{7 / 2}$ in Fig. 11 is not yet correct since we have not taken the Fermi function into account. It is best to consider the amplitude dependence of the $4 f_{5.2}$, or the SO peak, in Fig. 11 since this is not complicated by the Fermi function. Now we see that peak height analysis and integration yield very similar results. Indeed, we find that there is almost no temperature dependence above $T_{v}$, with all the temperalure dependence below $T_{1}$, being due to the phase transition itself and not to SIM phenomena.

It is important to note that we are seeing the bulk transition in the $4 f_{5,2}$ peak, so that the idea of a sub-surface with a single $T_{K}$ larger than the bulk, is of necessity eliminated. It was a preposterous idea in any case.

The high resolution of the data and narrow lineshapes of the $\mathrm{YbInCu}_{4}$ data allow more comparisons to SIM. In Fig. 12 we overlay the $20 \mathrm{~K}$ and $200 \mathrm{~K}$ spectra after removing the surface and non-f contributions via the background shown in Fig. 10. We have already pointed out (Joyce et al., 1998) that the $200 \mathrm{~K}$ spectral weights are at least an order of magnitude too large relative to SIM predictions. But even more information is to be obtained. By normalizing the $200 \mathrm{~K}$ spectrum at the SO peak height of the $20 \mathrm{~K}$ spectrum we find that the $200 \mathrm{~K}$ data is actually substantially broader than the $20 \mathrm{~K}$ data. A convolution with a $90 \mathrm{mcV}$ Gaussian is necded to make the $20 \mathrm{~K}$ FWHM equal to the $200 \mathrm{~K}$ FWHM. This is exactly opposite to SIM predictions where a line narrowing of an order of magnitude is needed for 
lïg. 12. Divalent bulk $4 f$ peaks at $20 \mathrm{~K}$ and $200 \mathrm{~K}$ after subtracting off background. Thin-lined spectrum is $200 \mathrm{~K}$ spectrum normalized at peak of $20 \mathrm{~K}$ SO intensity. Note that $200 \mathrm{~K}$ spectrum is broader than $20 \mathrm{~K}$ spectrum, contrary to SIM expectations where thermal broadening is not included.

the $200 \mathrm{~K}$ data. On the other hand, the $90 \mathrm{meV}$ thermal broadening is almost exactly what we: : have becn claiming all along for the $4 \mathrm{f}_{7 / 2}$ fcaturc in $\mathrm{Yb}$ compounds, and the $4 \mathrm{f}_{5,2}$ fcature in $\mathrm{Cc}$ compounds. Whether this broadening is due to phonons or to PAM effects is not delineated, but it is a fact of life.

To sum up, it is clear that in $\mathrm{YbInCu} 4$ we are seeing true bulk phenomena along with surface features. The measured $n_{f}$ for the low- $T_{K}$ region (above $T_{V}$ ) is $\approx 0.5$ vs the expected 0.9 , the spectral weight is at least an order of magnitude too large, there is no peak shift at the phase transition where a $40 \mathrm{meV}$ shift is predicted, thermal broadening effcets go opposite to SIM predictions but are precisely in line with our previous observations. Analysis of the $4 \mathrm{f}_{5 / 2}$ peak is preferred to the $4 \int_{\tau_{2}}$ owing to no interference from the Fermi function.

\section{Momentum Dependent Effects}

Perhaps the strongest argument in favor of a model which includes the periodic lattice is the observation of momentum dependent effects at temperatures far above the Kondo temperature. The PAM allows for such an electronic structure. By contrast, within GS and NCA the KR and its sidebands are a dispersionless feature [Bickers et. al., 1987], indeed a thermodynamic quantity of a material, whose properties (i.e., the width, position in energy, spectral weight, etc.) are essentially determined by the value of $T_{K}$. True enough, at low temperatures it is agreed that there is band formation once coherence sets in (after all, the de Haas-tan Alphen effect has been observed by numerous times ([Lonzarich [1988], Springford and Reinders [1988]), but the dispersion must be of the order $T_{K}$, and then only at $T<<$ $T_{K}$, or below the so-called coherence temperature. The manifestation of angle dependent effects, particularly if these exist at $T \gg T_{K}$, then, would represent a serious disagreement with NCA.

$\mathrm{CePt}_{2+\pi}$ Amplitude Modulations: Just such angle dependent effects have been reported by Andrews et. al first in $\mathrm{CePt}_{2+x}(0<\mathrm{x}<1)$ [Andrews et. al., 1995a], and later in $\mathrm{CeSb}_{2}$ [Andrews et. al., 1995b] and $\mathrm{CeBe}_{13}$ [Andrews et. al., 1996]. The data for $\mathrm{CePt}_{2+\mathrm{x}}$ are shown in Fig. 13 where the amplitude of the near- $E_{\mathrm{F}}$ feature is seen to undergo periodic amplitude modulation, the period of which coincides exactly with the inverse lattice. The insets indicate the positions in the lattice (surface projection of the Brillouin zone) where the various spectra were taken, while the angles are measured with respect to the surface normal (i.c., the $\Gamma$-point). Very large effects are observed, which bring into question the thermodynamic nature of the near- $E_{\mathrm{F}}$ feature. The data were taken at resonance (photon energy $=120$ $\mathrm{cV}$ ) at a tempcrature of $120 \mathrm{~K}$ using a VSW HA50 angle resolved analyzer with a $2^{\circ}$ acceptance cone and about $90 \mathrm{mcV}$ energy resolution. The data is highly reproducible. 
Fig. $12 \mathrm{CePt}_{2+x}$ ARPES spectra at the indicated point in the Brillouin zone. Note that the $\mathrm{H}_{52}$ amplitude shows amplitude variations exactly periodic with Brillouin zone.

The $\mathrm{CePt}_{2+\mathrm{x}}$ sample was prepared in-situ by evaporating Ce onto a $\mathrm{Pt}$ (111) single crystal. Analysis with LEED and XPS [Tang et. al., 1993] as well as comparison of $\mathrm{Pt}-4 \mathrm{~d}$ intensities of the in-situ grown specimen to arc melted poly-crystals, determined the composition to be most likely $\mathrm{CePt}_{2.2}$, with the value of $\mathrm{x}$ determined to within $0<\mathrm{x}<1$. Bulk propcrty measurements on are melted specimens with compositions between $\mathrm{CePt}_{2}$ and $\mathrm{CePt}_{3}$ indicated very similar properties throughout the composition range so that the inexact determination of $\mathrm{x}$ is irrelevant to the physics. The Kondo temperatures were found to be less than $20 \mathrm{~K}$ for all compositions, with the magnetic susceptibility showing Curie-Weiss behavior down to $20 \mathrm{~K}$. From bulk property measurements one would conclude that the materials are ncarly trivalent so that it is all the more surprising that such an intense $K R$ is observed at $T=$ $120 \mathrm{~K}$.

It is significant that the amplitude modulation effects are seen primarily in the KR. Indeed, in the figure the data have been normalized to the peak value of the spin-orbit peak, the $\mathrm{Ce}-4 \mathrm{t}_{7}$. Because the amplitude modulation is seen primarily in the $\mathrm{KR}$ it rules out phothelectron diffraction as the source of the amplitude effect, since the orbital symmetry and the atomic point of origin of both features is identical, while the kinetic energies of the photremitted clectrons from the two features are likewise ncarly identical. From photoclectron diffraction both peaks would be expected to undergo similar amplitude modulation. Moreover, photoelectron diffraction effects are not necessarily periodic with the inverse lattice, but are rather a geometric effect [Fadlcy, 1987] which yiclds maximum amplitude from forward scattering around masking atoms near the surface (the forward flashlight). The observation of dispersion in some systems in any case points to Bloch states.

In their first paper on the subject Andrews ct. al. [1995a] interpreted the amplitude effect as resulting from the dispersion of an extremely narrow band above $E_{\mathrm{F}}$. They showed that the effect can easily be accounted for if a Lorentzian feature, derived from a very flat band about $20 \mathrm{meV}$ below $E_{\mathrm{F}}$, disperses to just above the $E_{\mathrm{F}}$. The dispersion itself was not observable owing as much to the large natural linewidth $(\approx 100 \mathrm{meV})$ as to instrument reso- 
lution. In subsequent materials studied there appears to be actual evidence for dispersion (see below) but surprisingly in a direction opposite to that assumed by Andrews et. al. (1995al.

The temperature at which the measurements were taken $\left(T_{m}=120 \mathrm{~K}\right)$ is a most important factor in solving the heavy fermion puzzle. For $\mathrm{CePt}_{2+x}$ we see that the measuring temperature is very high, $6 \mathrm{~T}_{\mathrm{K}}<\mathrm{T}_{\mathrm{m}}<10 \mathrm{~T}_{\mathrm{K}}$. Based on NCA, the KR and any possible sidebands should not even exist at $10 \mathrm{~T}_{\mathrm{K}}$, much less be exhibiting Bloch state behavior which should hate becn obseriable only at $T_{m} \ll T_{K}$

Fig. 14 Digitized and replotted data of Garnier et al. (1997) which strongly suggest (left panel) dispersion of both the $+f_{5 i 2}$ and $\mathcal{f}_{7 / 2}$ features. Arrows indicate apparent peak positions. The double arrow for $\theta=6^{\prime \prime}$ derives from the uncertanity of the peak position. The right panel is a blowup of the near- $\mathrm{E}_{\mathrm{F}}$ region.

Observation of Dispersion: Superb confirmation of the above results was reportcd in a recent publication (Garnicr, ct al., 1997) in which the reported resolution was 5 $\mathrm{meV}$ and the data were taken at $40.8 \mathrm{eV}$ photon energy. While the authors reported that no dispersion was evident, a replotting of the data in Fig. 14 suggests that one could reach different conclusions. Indeed, if the feature at $-100 \mathrm{meV}$ in the $\theta=6^{\circ}$ spectrum can be considered as $4 f$-derived, then there exists about $100 \mathrm{meV}$ of dispersion. The double arrow on this feature at $\theta=6^{\circ}$ signifies the uncertanity of the exact peak position, but irrespective of the exact position it is hard to avoid the conclusion that the peak disperses. Indeed, not only the $4 f_{52}$ but also the $4 f_{7.2}$ is sees to strongly disperse in the left panel of Fig. 14. A blowup of the $4 f_{5.2}$ dispersion is shown in the right panel. The observed dispersion is surprisingly close to the predictions of the PAM which suggest that the $4 \mathrm{f}$ band crosses $E_{\mathrm{F}}$ away from a symmetry direction and loses $f$-character as it disperses below $E_{\mathrm{F}}$. In Fig. 14 the most intense $4 \mathrm{f}$ peak is obtaincd at $\theta=2^{\circ}$ (away from (111)) and is nearly totally attenuated at $\theta=6^{\circ}$ where it is found below $E_{F}$. At normal emission the $4 f_{32}$ intensity is consistent with a peak just above $E_{F}$ All these observations are entirely consaistent with the PAM predictions in which $4 f$-band dispersion is much larger than $T_{K}$, persist to very high temperatures, and crosses $E_{F}$ away from symmatry points in a cubic lattice.

Dispersion in CeSb2: Amplitude modulation of the Ce-4f $f_{s / 2}$ is seen to some degree in nearly every single crystal Ce heavy fermion investigated, but direct evidence for dispersion requires better energy and momentum resolution. An especially crucial feature is a mirror-like cleaved surface without which all dispersion can be washed out. The intensity of the $\mathrm{Ce}-4 \mathrm{f}_{5,2}$ as well as the strength of the amplitude modulations correlates more strongly with crystalline perfection than with $T_{K}$, with the most perfect single crystals yielding the most intense KR's. Indeed, an intense Ce- $4 f_{5 ; 2}$ peak is observed in $\mathrm{CeSb}_{2}$, a layered material with a ferromagnetic transition at $10 \mathrm{~K}$ which implies that $T_{K}<10 \mathrm{~K}$. Amplitude modulation was first reported by Andrews et al. (1994). Subsequently (Arko et al., 1997), data at lower 
Fig. $15 \mathrm{CeSb}_{2}$ ARPES spectra along two directions in the Brillouin zone, but both starting at (001). In (a) the dispersion is below $F_{F}$ and the $+f$ intensity rapidly diminishes as the band takes on d-character. In (b) the dispersion is above $E_{F}$ with only minor diminnution of $+f$ intensity, consistent with the PAM.

encrgics and $\mathrm{T}=20 \mathrm{~K}$ revealcd about $25 \mathrm{mcV}$ of dispersion, with bchavior amazingly similar to that found in Fig. 14. The left panel of Fig. 15 shows ARPES data at $45 \mathrm{eV}$ photon cncrgy, and ite sec that as the peak disperses away from $E_{\mathrm{F}}$ it rapidly loses intensity as the fcharacter is diminished and replaced by d-character (according to the PAM). On the other hand, if the dispersion is toward $E_{F}$ (Fig. $41 \mathrm{~b}$ ), the $4 f_{5 / 2}$ intensity is only slightly diminished as the $4 \mathrm{f}$ band disperses just above $\mathrm{E}_{\mathrm{F}}$ and flattens out, leaving the tail on the occupied side. This tlattened region can be identified with the KR as in the SIM, though it persists far above $T_{K}$. shown here.

Similar dispersion has becn obscrved also in $\mathrm{CcBc}_{13}$ (Andrew's ct al., 1996) but is not

\section{Uranium Compounds}

Measurements on highly correlated single crystal uranium compounds again seem to point to a band nature of $5 f$ electrons at $T \gg T_{K}$. Indications of this were already evident in carly publications of Arko et. al. [1983] in an angle resolved PES study of $\mathrm{UIr}_{3}$ where evidencic for slight dispersion of $5 \mathrm{f}$ states at $300 \mathrm{~K}$ was presented for the first time. In that paper dispersion was not considered unusual however, since, just as in $\mathrm{URh}_{3}$, dHvA measurements [A rko et. al., 1975, 1976] indicated a rather normal, transition-metal-like material with clfective masses again no larger than 5 to 10 . More intriguing were the indications of periodic ARPES - tructure [Arko et. al., 1990] in the 5t features of the heavy fermion material $\mathrm{UPt}_{3}$ measured at $300 \mathrm{~K}$. Although in $\mathrm{UPt}_{3}$ the near- $\mathrm{E}_{\mathrm{F}}$ 5f feature never showed measurable dispersion, it, width was resolution limited $(\approx 200 \mathrm{meV})$ while it's intensity vanished near the $\mathrm{M}$-point in the hexagonal lattice. Thus knowledge of the existence of sharp $5 \mathrm{f}$ features having intensities periodic with the inverse lattice in uranium compounds even at $300 \mathrm{~K}$ was available some time ago, but this information was mostly overlooked.

Recently single crystal uranium compounds have been investigated [Arko et. al., 19981 both at the resonant photon energy as well as at much lower photon energies where momentum and encrgy resolution is substantially better. Evidence for dispersion is again found, primarily in materials having the most perfect cleaved surfaces. Here we will concentrate on $\mathrm{USb}_{2}$, an antiferromagnet at $200 \mathrm{~K}$, and $\mathrm{UPt}_{3}$, a heavy Fermion with $\mathrm{T}_{\mathrm{K}} \approx 10 \mathrm{~K}$. Very recently $50 \mathrm{mcV}$ of dispersion was also found in UAsSe, a material claimed to display non-Fermi-liquid behavior, but this data is still too fresh to include here. All data shown were collectad at $T \approx 20 \mathrm{~K}$, al though data at higher temperatures exists.

In Fig. 16 we present ARPES data for USb, at lower photon encrgics. This material cxhibits a layered tctragonal structure which is ideal for ARPES since the two-dimensional 
Fig. 16 ARPES spectra from a (100) surface of $\mathrm{LSb}_{2}$ along the $\Gamma-\mathrm{X}$ diraction. Peaks $\mathrm{A}$ and $B$ are $5 \mathrm{f}$-derived, based on $\mathrm{h} v$-dependence.

nature allows for band mapping without the complication from the third dimension. ARPES data at $h v=30 \mathrm{cV}$ and $\mathrm{T}=20 \mathrm{~K}$ arc shown as a function of angle [Arko ct. al., 1997] along: the $\Gamma$-X direction. Two dispersive peaks labeled $A$ and $B$ are evident. Both peaks are unambiguously $5 f$ derived based on the photon energy dependence of the intensity, but peaks $B$ appear to have substantial 6d-admixturc, particularly as the binding cnergy increases. The dispersion of peak $\mathrm{B}$ is obvious and clearly $5 \mathrm{f}$ in nature, based on photon energy dependence. Indeed, this represents the first clear and unambiguous evidence of $5 \mathrm{f}$ dispersion.

The dispersion of peak A is smaller, of the order of $30 \mathrm{mcV}$, though it is not obvious from Fig. 16. Indeed, peak A is nearly absent near $\Gamma$. Interestingly, band A disperses below $E_{p}$ as its intensity decreases, which is similar to the effect seen in Ce heavy fermions above. This sharp near- $E_{\mathrm{F}}$ feature shows very little temperature dependence (except for Fermi function convolution), similar to the situation found in $\mathrm{Ce}$ and $\mathrm{Yb}$ compounds.

1Fig. 17 ARPIS spectra from $\mathrm{CP}_{3}(001)$ at $\mathrm{T}=20 \mathrm{~K}$, taken at hv $=40 \mathrm{el}\left(\mathrm{T}_{\mathrm{K}} \approx 10 \mathrm{~K}\right)$. Four dispersive peaks are evident. Note the vanishing of peak $\mathrm{A}$ between 2 and 3.5 as it disperses above $F_{\Gamma}$. 
Dispersion in $\mathbf{U p t}_{3}$ : Since USb $\mathrm{US}_{2}$ is an anti-ferromagnet below $20(0 \mathrm{~K}$ it leares some room for argument regarding the band nature of heary fermions despite the fact that one normally cxpects a magnetic state to exhibit greater localization. The next logical step is to look for cridence of dispersion and $5 f$ band states in uranium compounds which, unlike antiferomagnetic $\mathrm{USb}_{2}$, are unambiguously heavy fermions - c.g., UPt ${ }_{3}$.

LPt, (6) 1 ) was studied at lower photon energies, at temperatures of $20 \mathrm{~K}$ and $80 \mathrm{~K}$, and the results are highly suggestive of dispersion of the near- $E_{\mathrm{F}}$ peak as seen in Fig. 17. Here the data w ere taken at hv $=40 \mathrm{eV}$, resolution $\Delta \mathrm{E}=40 \mathrm{meV}$, and different angles relative to $\Gamma$, i.c., the surface normal in the Brillouin zone. . There is an obvious amplitude effect just as in Ce compounds. Note the complete vanishing of the near- $E_{F}$ peak between $2^{\circ}$ and $3.5^{\circ}$ analyzer angle. The drop in intensity corresponds reasonably well with band calculations which show a narrow band, located preciscly at $E_{F}$ at the $\Gamma$-point, dispersing above $E_{F}$ at about $0.3 \AA^{-1}$, or, just about $5^{\circ}$ from $\Gamma$. The peak at $2^{\circ}$ from $\Gamma$ is dispersing toward the Fermi energy. The peaks beyond $5^{\circ}$ are then probably due to different bands. The inset emphasizes the dispersion between the $1^{\circ}$ and $25^{\circ}$ data. It is not certain that improved resolution will be able to separate out the additional bands since the natural linewidths may be larger than the band separations, but it is nevertheless an experiment worth doing. Data taken at $80 \mathrm{~K}$ essentially reproduces the $20 \mathrm{~K}$ data. This data clcarly shows that most of the LDA-derived band structure exists already above $T_{K}$, except that the $f$-derived bands are flatter than predicted. This is totally consistent with the PAM predictions.

\section{CONCLUSIONS}

Nearly every recent measurement performed on $\mathrm{Ce}$ or $\mathrm{U}$ based single crystal heavy fermion compounds sccms to point to the cxistence of narrow f-clectron bands for a wide range of temperatures. Yb compounds are less clear but are not totally inconsistent with this : idea. The clearest evidence for bands is in uranium systems where actual dispersive bands have becn observed in $\mathrm{USb}_{2}$ and UPt $\mathrm{U}_{3}$, as wcll as suggestions of dispersion in $\mathrm{UBe}_{13} . \mathrm{Vcry}$ recently $50 \mathrm{meV}$ of $5 \mathrm{f}$ dispersion has also been found in UAsSe. In Ce compounds dispersion has been observed in $\mathrm{CePt}_{2+\mathrm{x}}, \mathrm{CeSb}_{2}$, nad $\mathrm{CeBe}_{13}$, at temperatures far above $\mathrm{T}_{\mathrm{K}}$. The fcaturc commom to all these compounds is a mirror-like clcaved surface. The lcast obvious are $\mathrm{Yb}$ compounds where the $4 \mathrm{f}$ PES features for all intents and purposes are indistinguishable from core levels pinned at the Fermi energy, but with complications of surface states at lower photon encrgics. Some very recent data on $\mathrm{YbCdCu}_{4}$, not included in this paper, does actually provide the first hint of dispersion even in $\mathrm{Yb}$ compounds so that it appears that all system may yet show some universal behavior. No mirror surfaces have been obtained in $\mathrm{Yb}$ compounds. In all cases the $\mathrm{n}_{\mathrm{f}}$ is far too low relative to SIM predictions.

By contrast, few systems unambiguously exhibit the behavior predicted by the single impurity model. While it is possible to fit a particular spectrum within NCA by adjusting parameters, the lack of systematics with $T_{K}$ are glaring. In particular, there is no clear systematic scaling of the near- $E_{F}$ spectral weight with $T_{K}$, the width of the $f^{0}$ peak is approximately constant with $T_{K}$, there is no temperature dependence which cannot be explained by conventional effects of thermal broadening and Fermi function effects (here, perhaps, the broadening may be attributed to the PAM), the spin-orbit feature does not shift toward $E_{F}$ as $T_{k}$ increases, and no clear-cut observation of a crystal field state has been reported despite sulficient resulution. Indecd, the temperature dependence in $\mathrm{Ce}$ compounds does not provide cridence for the existence of an intense $4 \mathrm{f}$ DOS feature just above $E_{\mathrm{F}}$, otherwise one would obscric a shift of the near- $E_{\mathrm{F}}$ peak toward the Fermi cnergy (vs the observed shift in the (opposite dircetion) as was first predicted by Gunnarsson and Schonhammer [1986]. In Yb compounds the spectral weight of the near- $E_{\mathrm{F}}$ peaks (i.e., the $n_{\mathrm{f}}$ ) is so totally inconsistent with SIM predictions as to be sufficient by itself to seriously question the model. The theory is only valid down to $n_{f}$ values of about 0.75 , while typical $n_{f}$ 's in Yb compounds are measured at 0.65 even for $T_{K}$ 's of $35 \mathrm{~K}$. If the existence of the Kondo resonance with the stated properties is a necessary consequence of the SIM, as appears to be the case, then we must look beyond SIM to understand heary elcetron behavior.

One the other hand, thermodynamic data are in better agreement with the predictions of SIM, with the recent bandwtdth extractions being the only serious disagreement. There is a consistent relationship between $T_{K}$ and, say, the peak in the magnetic susceptibility; the specific heat $\gamma$, and inelastic neutron scattering results. Actually, the most important parameter is in fact the hybridization from which $T_{k}$ and all other parameters follow within SIM. This has presented a dilemma prior to the emergence of new theories, many of which build 
on the SIM. Any successful theory must simultaneously be able to correctly predict the microsecopic as well as the macroscopic properties. SIM and its extensions appear primaril! successful with the latter, although, again as pointed out, the bandwidths obtained from a SIM analyis of susceptibility data in $\mathrm{YbXCu}_{4}$ compounds are totally unphysical $(0.1 \mathrm{eV}$ is measured values of $\approx 10 \mathrm{cV}$.). Nerertheless, at some lerel NCA contains some truth. Elcetron-clectron correlations are indeed important, and formation of the singlet state with conduction clectrons is not in dispute (except in the model of Liu (1993, 1997)). Somehow this must be reconciled with the existence of $f$-bands far above $T_{K}$ which are by clearly obsericd in ARPES measurements.

Various renormalized band approaches [Zwicknagel, 1992; Strange and Newns 1986] have succecded in cffectively' reproducing the large clectron masses in heary fermions, but they are not in general able to accommodate the numerous magnetic states as wcll as superconductivity within the same model. A rery interesting model that has been recently proposed is the two-clectron band approach of Sheng and Cooper [1995], in which correlations are first included cxplicitly between the $f$-electrons and ligand conduction band states ( $f$ $l$ correlations) while $f$ - $f$ correlations are treated within an infinite- $U$ approximation. While this is quite similar to the SIM approach, it differs in that a collective response of the ligand clectrons which yields the Kondo resonance is not included. Instead, the singlet (or triplet) state is formed as an $f-l$ pair of clectrons which then form paired band states.

The nascent studies of the Periodic Anderson Lattice Model, do contain some of the ingredients crucial to the description of the photo-emission spectrum, such as substantially more intensity in the $f^{1}$ peaks vs the $f^{0}$ peak thus yiclding much smaller $n_{f}$ values, the formation of dispersive quasi-particle bands, a much more mild temperature dependence than found in the SIM, broadening wich was initially attributed to phonons, and the weak hybridization at $E_{F}$ yielding flat bands at $E_{F}$ while strong hybridization away from $E_{F}$ allows $f$-spectral: weight to disperse to encrgies far larger than $\mathrm{kT}_{\mathrm{K}}$ (as observed).

It is, of course, a long road from the first inception of any model to the final detailed proof of its applicability. In its early stages it appears to qualitatively fit the ARPES data, including the mild temperature dependence. Final proof - or discarding - of this theory, however, will occur only after successful - or unsuccessful - attempts to fit real spectra.

\section{Acknowledgements.}

We rould like to acknowledge useful conversations with J. Allen, J. Bonca, Barry Cooper, A. Chattopadhyay; D.L. Cox, P. van Dongen, J.K. Freericks, B. Goodman, M. Grioni, D.W. Hess, M. Hettler, H.R. Krishnamurthy, Jon Lawrence, D. McLaughlin, Th. Pruschke, R.N. Silver. Much of the cxperimental work was performed under the auspices of the U.S. Department of Energy, Office of Basic Energy Sciences, Division of Materials Science. Measurements were performed at the Los Alamos U3C beamline at the NSLS and at the Synchrotron Radiation Center of the Univ. of Wisconsin.

\section{References}

Allen, J.W., 1992, Synchrotron Radiation Research:Advances in Surface and Interface Science. Volume I: Techniques, edited by Z. bachrach, Plenum Press, New riork.

Allen, J.W. S.-J. Oh, O. Gunnarson, K. Schonhammer, M.B. Maple, M.S. Torikichrili and I. Lindau, 1986, Adrances in Physics 35, 275.

Anderson, P.W., 1961, Phys. Rev. 124, 41.

Andres, K., J.E. Graebner and H.R. Ott, 1975, Phys. Rev. Lett. 35, 1779.

Andrews, A.B., J.J. Joyce, A.J. Arko, J.D. Thompson, J. Tang, J.M. Lawrence, and J.C. Hemminger, 1995a, Phys. Rev. B 51, Rapid Comm., 3277.

Andrews, A.B., J.J. Joyce, A.J. Arko, J.D. Thompson, Z. Fisk, J. Tang, J.M. Lawrence, P. S. Riseborough, and P.C. Cantield, 1995b, Physica B 206 \& 207, 83.

Andrew's, A.B., J.J. Joyce, A.J. Arko, Z. Fisk, and P.S. Riseborough, 1996, Phys. Rev. $B \leq 53,3317$.

Andrews, A.B., R.I.R. Blyth, A.J. Arko, J.J. Joyce, Z. Fisk, J.D. Thompson, R.J. Bartlett, P.C. Cantield, C.G. Olson, P. Benning, and P.S. Riseborough, 1994, Phýsica B 199\& 200, 15.

Arko, A.J., J.J. Jovce, A.B. Andrews, R.I.R. Blyth, C.G. Olson, P.C. Canfield, P.J. Benning, D.M. Poirier, J.H. Weaver, and P.S. Riseborough, 1994, in Strongly Cor- 
related Electronic Materials, edited by K. Bedell, S.-Y. Huang, A. Balatsky, D.E.

Melter, and E. Abrahams, (Aduison Wesley), pg. 101.

Arko, A.J. D.D. Koelling, and B. Reihl, 1983, Phys. Rev. B 27, 3955.

Arko, A.J., J.J. Soyce, A.B. Andrews, J.D. Thompson, J.L. Smith, D. Mandrus, M.F. Hundles A.L. Cornclius, E. Moshopoulou, Z. Fisk, and P.C. Canficld, Alois Menoriky. 1996. Phys. Rev. B 56, Rapid Com., R7041.

Arko. A.J., J.J. Joyce, A.B. Andrews, J.L. Smith, J.D. Thompson, Z. Fisk, E. Mishopoulou. A. Menorsky and P.C. Canficld, 1997, Physica B 230-232, 16.

Arko, A.J. J.J. Josce, L.E. Cox, L. Morales, J. Sarrao, J.L. Smith, Z. Fisk, A. Menorsky, A. Tahvildar-Zadeh, and M. Jarrell, 1998, J. Alloys and Comp.

Arko, A.J., J.J. Joyce, P.C. Canficld, J.D. Thompson, R.J. Bartlett, Z. Fisk, J. Lairrence, J. Tang, and P. Riseborough, 1993, in Vacuum Ultraviolet Radiation Physics, cdited by F.J. Wuillemier, Y. Petroff, and I. Nenner, (World Scientific: Singapore), p. 291.

Arko, A.J., M.B. Brodsky, G.W. Crabtree, D. Karim, D.D. Koelling, L.R. Windmiller, and J.B. Ketterson, 1975, Phys. Rev. B 12, 4102.

Arko, A.J., M.B. Brodsky, G.W. Crabtrce, D. Karim, L.R. Windmiller, and J.B. Ketterson, 1976, in Plutonium 1975 and other Actinides, H. Blank and R. Linder, cds. (North Holland, Amsterdam) p. 325.

Arko, A.J., P. Armstrong, and M. Wire, 1990, Physica B163, 167.

Bickers, N.E., 1987, Rev. Mod. Phys. 59, 845.

Bickers, N.E., D.L. Cox and J.W. Wilkins, 1985, Phys. Rev. Lett. 54, 230.

Bickers, N.E., D.L. Cox and J.W. Wilkins, 1987, Phys. Rev. B 36, 2036.

Blyth, R.I.R., J.J. Joyce, A.J. Arko, J. Tang, and J.M. Lawrence, 1992, Phys. Rev. Lett.69, 3418.

Blyth, R.I.R., J.J. Joyce, A.J. Arko, P.C. Canficld, Z. Fisk, J.D. Thompson, R.J. Bartlett, J. Tang, and J.M. Lawrence, 1993, Phys. Rev. B 48, 9497.

Fadley, C.F., 1987, Physica Scripta T 17, 39.

Fisk, Z., J.L. Sarrao, J.L. Smith, and J.D. Thompson, 1995, Proc. Natl. Acad. Sci. USA, 92, 6663.

Galera, R.M., A.P. Murani, and J. Pierre, 1989, Physica B 156\& 157, 801.

Garnicr, M., D. Purdic, K. Breuer, M. Hengsberger, and Y. Baer, 1997, Phys. Rcv. B 36. R11399.

Grewe, N.and F. Steglich, 1991, Handbook on the Physics and Chemistry of the Rare Earths, K.A. Gschncidncr, Jr. and L. Eycrling, cditors, (North-Holland, Amsterdam), Vol. 14, pp. 343-474.

Grioni, M., D. Malterre, P. Weibel, B. Dardel, and Y. Baer, 1993, Physica B 188, 38.

Gunnarson, O., and K. Schonhammer, 1985, Phys. Rev. B 31, 4815.

Gunnarson, O., and K. Schonhammer, 1986, in Theory of Heavy Fermions and Valence Fluctuations, T. Kasuya and T. Saso, editors (Springer-Verlag, Berlin, 1986) p. 110.

Hess, D.W.. P.S. Riseborough, and J.L Smith, 1993, Encvclopedia of Applied Physics, Vol. 7, George L. Trigg., ed., (VCH Publishers, NY), pp.435-463.

Hirsch, J.E. and R.M. Fye, (1989), Phys. Rev. Lett. 56, 2521.

Hulner. S. 1986, J. Phy's. F 16, L31.

Hutner, S, 1992, Z. Phys. B 86, 241.

Hulner, S. L. Schlapbach, 1986, Z. Phys. B 64, 417.

Jarrell. M. and J.E. Gubernatis, 1996, Physics Reports, Vol. 269, No.3, p133-195.

Joyce J.J., and A.J.Arko, 1993a, Phys. Rev. Lett. 70, 1181.

Jojec, J.J., A. J. Arko, J. M. Lawrence, P. C. Canfield, Z. Fisk, R. J. Bartlett, and J. D. Thompson. 1992c, Phys. Rev. Lett. 68236.

Joyce, J.J., A. J. Arko, J. M. Lawrence, P. C. Canfield, Z. Fisk, R. J. Bartlett, J. D Thompson, and J. L. Smith, 1992a, J. Less Comm. Mct., 181, 161.

Joyce, J.J., A.B. Andrews, A.J. Arko, R.I.R. Blyth, R.J. Bartlett, C.G. Olson, D.M. Poirier, P.C. Canfield, and P.J. Benning, 1996, Phys. Rev. B., to be published.

Joyce, J.J., A.J. Arko, A.B. Andrews, and R.I.R. Blyth, 1994a, Phys. Rev. Lett. 72, 1774

Joyce, J.J., A.J. Arko, A.B. Andrews, R.I.R. Blyth, R.J. Bartlett, J.D. Thompson, Z. Fisk, P.S: Riscborough, P.C. Canficld, C.G. Olson, and P.J. Bcnning, 1994b, Physica B 205, 365. 
Joyce, J.J., A.J. Arko, J.L. Sarrao, K.S. Graham, Z. Fisk, and P.S. Riscborough, 1998. submittat to Phys. Rev. Letters.

Joyce. J.J. A.J Arko, J.M. Lawrence, P.C. Cantield, R.J. Bartlett, Z. Fisk, and J.D.

Thompion. J. Tang, 1992b, Solid State Comm. 83, 551.

Joyec, J.J. A.J. Arko, P.S. Riscborough, P.C. Canficld, J.M. Lawrence, R.I.R. Blyth, R.J. Burtlatl, J.D. Thompson, and Z. Fisk, 1993b, Physica B 186-188, 31.

Kakizaki, A.. A. Harasawa, T. Ishii, T. Kashiwakura, A. Kamata, S. Kunii, 1995, J.

Phis. Soc. Japan 64, 302.

Kang. J.S., J.W. Allen, O. Gunnarsson, N.E. Christensen, and O.K. Anderson, Y. Las-

sailly, M.B. Maple and M.S. Torkachvilli, 1990, Phys. Rev. B 41, 6610.

Larr rence, J.M., and D.M. Mills, 1991, Comments Cond. Mat. Phys., 15, 163.

Lawrence, J.M., G.H. Kwei, P.C. Cantield, J.G. DeWitt, and A.C. Lawson, 1994 Phys. Rev. B49.

Lec, P.A., T.M. Rice, J.W. Serene, L.J. Sham, and J.W. Wilkins, 1986, Com. Cond. Mat. Phys. $12,99$.

Liu, L.Z., J.W. Allen, O. Gunnarsson, N.E. Christensen, and O.K. Anderson, 1992, Phys. Rev. B 45, 8934.

Liu, S.H., 1993, in Handbook on the Physics and Che" istry of Rare Earths, Vol.

17, Ch. 111 (North Holland, Amstcrdam, 1993) pp 87-148.

Liu, S.H., 1997, Physica B 240, 49

Lonzarich, G.G., 1988, J. Magn. Magn. Mat. 76-77, 1.

Malterre, D., M. Grioni, P. Weibel, B. Dardel and Y. Bacr, 1992a, Europhys. Lett. $20,445$.

Malterre, D., M. Grioni, P. Weibel, B. Dardel and Y. Baer, 1992b, Phys. Rev. Lett. 68, 2656.

Matzdorf, R., G. Meister, and A. Goldmann, 1993, Surf. Sci. 286, 56.

Norman, M.R., 1985, Phys. Rev. B 31 , 6261.

Norman, M.R., D.D. Koclling and A.J. Frecman, 1985, Phys. Rev. B 31, 6251.

Nozieres, P., 1985, Ann. de Phys. $10,19$.

Oh, S.J., 1993, Physica B 186-188, 637.

Oliveira, L.N. and J. Wilkins (1981), Phys. Rev. Lett. 47, 1553.

Onuki, Y., 1993, Physica B 186-188, 92.

Ott, H.R., and Z. Fisk, 1987, in Handbook on the Physics and Chemistry of the Actinides, A.J. Frecman and G.H. Lander, editors, (North-Holland, Amsterdam), pp. 85-225.

Ott, H.R., H. Rudigier, Z. Fisk, and J.L. Smith, 1993, Phys. Rev. Lett. 50, 1595.

Patthey, F., B. Delley, W.D. Schneider, and Y. Baer, 1985, Phys. Rev. Lett. 55, 1518.

Patthey, F., J.-M. Imer, W.-D. Schneider, H. Beck and Y. Baer, 1990, Phys. Rev. B 42 , 8864.

Patthey, F., W.-D. Schncider, Y. Bacr, and B. Delley, 1987a, Phys. Rev. Lett. 5 8, 2810.

Patthey, F.. W.D. Schneider, M. Grioni, D. Malterre, and Y. Baer, 1993, Phys. Rev. Lctl. 70, 1179.

Patthes, F., W.D. Schneider, Y. Baer, and B. Delley, 1986, Phys. Rev. B 3 4, 2967.

Patthey, F. W.D. Schneider, Y. Baer, and B. Delley, 1987b, Phys. Rev. B 35, 5903.

Phys. Rev. Lett. 65, 496-9.

Pruschke. Th., and N. Grewe (1989), Z. Phys. B 74, 439.

Sheng. Q.G. and B.R. Cooper, 1995, Phil. Mag. Lett. 72, 123.

Silver, R.N., J.E. Gubernatis,D.S. Sivia, and Mark Jarrell, 1990

Springford, M., and P.H.P. Reinders, 1988, J. Magn. Magn. Mat. 76-77, 11.

Steglich. F., J. Aarts, C.D. Bredl, W. Lieke, D. Meschede, W. Franz, and H. Schafer, 1979. Phys. Rev. Lett. 43, 1892.

Steiner, M.M., R. C. Albers, and L. J. Sham, 1994, Phys. Rev. Lett, 7 2, 2923.

Stewart, G. R., 1984, Rev. Mod. Phys. 56, 755.

Stewart, G.R., Z. Fisk, J.O. Willis, and J.L. Smith, 1984, Phys. Rev. Lett. 52, 679.

Strange, P., and D. M. Newns, J. Phy's. F 16, 335.

Tahvildar-Zadeh, A.N., M. Jarrell and J.K. Freericks, 1997, Phys. Rev. B5 5, R3332.

Tahvildar-Zadeh, A.N., M. Jarrell and J.K. Freericks, 1998, In preparation.

Takahashi, T., T. Morimoto, T. Yokoya, M. Kohgi, and T. Satoh, 1994, Phys. Rer. B 49. 15688 .

Tang, J., J.M. Lawrence, and J.C. Hemminger, 1993, Phys. Rev. B 48, 15342. 
Thompson, J.D. and J.M. Lawrence, 1994, Handbook on the Physics and Chemistry of the Rare Earths, K.A. Gschneidner, Jr. and L. Eycrling, editors, (North-Holland. Amsterdam ). Vol 19, pp. 383-478.

Tjeng, L.H., S.-J. Oh, E.-J. Cho, H.-J. Lin, C.T. Chen, G.-W. Gweon, J.-H. Park, J.W. Allon, T. Suzuki, M.S. Makivic, and D.L. Cox, 1993, Phys. Rev. Lett. 71. $1+14$

Weibel, P. : M. Grioni, D. Malterre, B. Dardel, Y. Baer, and M. Besnus, 1993, Z. Phys. B 91, 3.37.

Weschke, E. C. Laubschat, T. Simmons, M. Domke, O. Strebel, and G. Kaindl, 1991. Phys. Rer. B 44, 8304.

Wilson, K.G., (1975), Rev. Mod. Phys. 47, 733.

Zwicknagl, Gertrud, 1992, Advances in Physics 41, 203. 


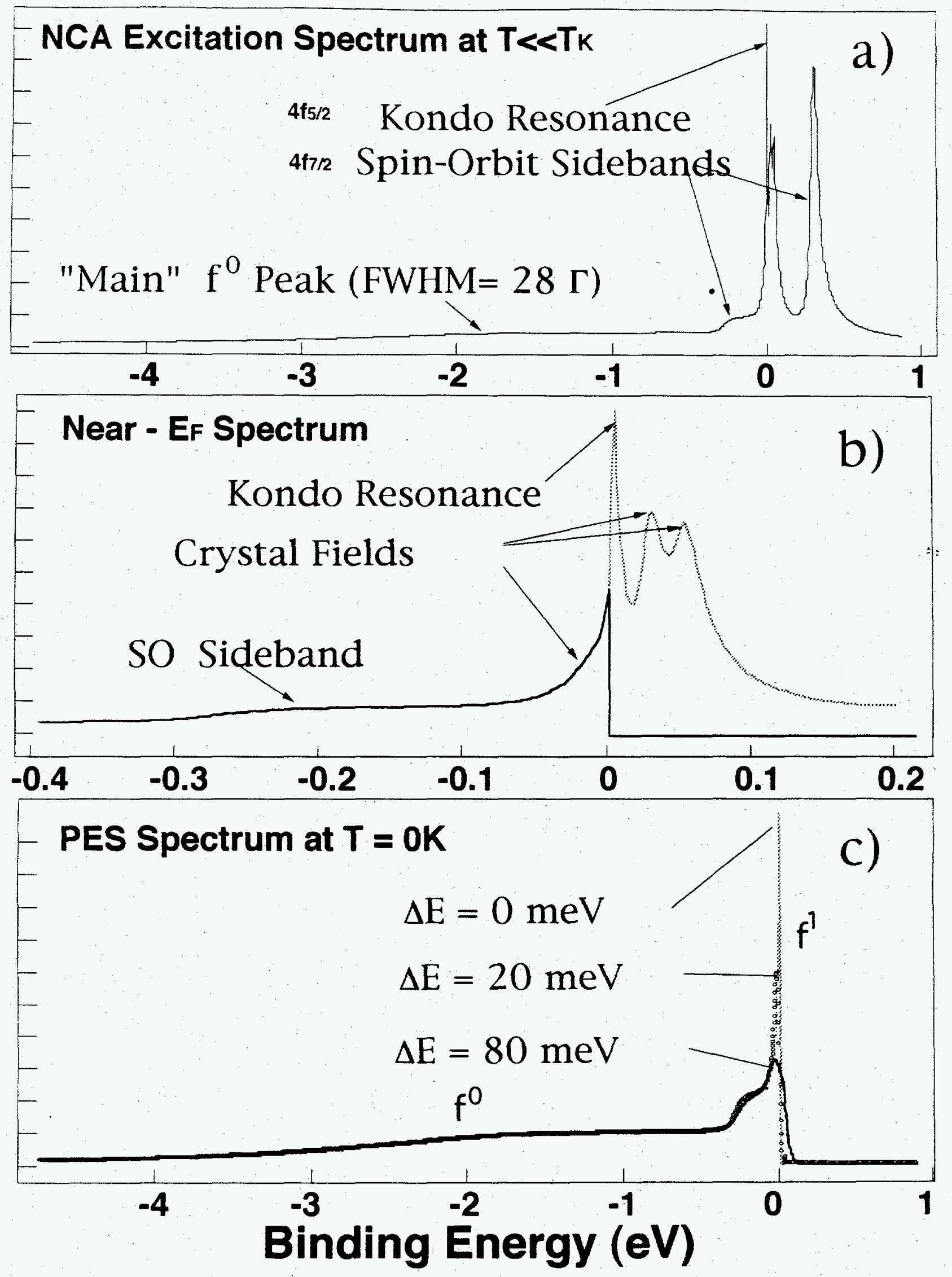

Fig. I 


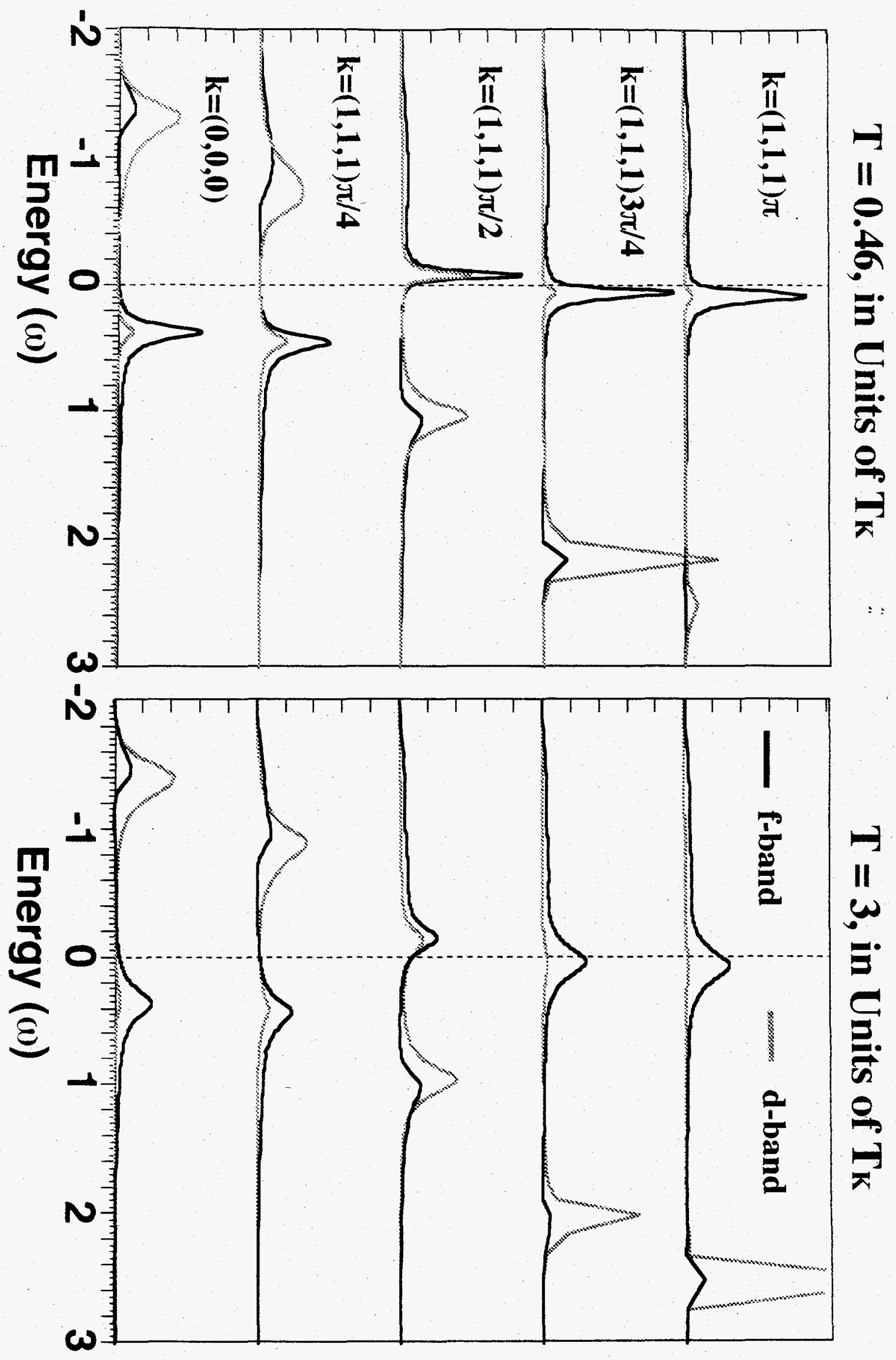

Fig. 2 


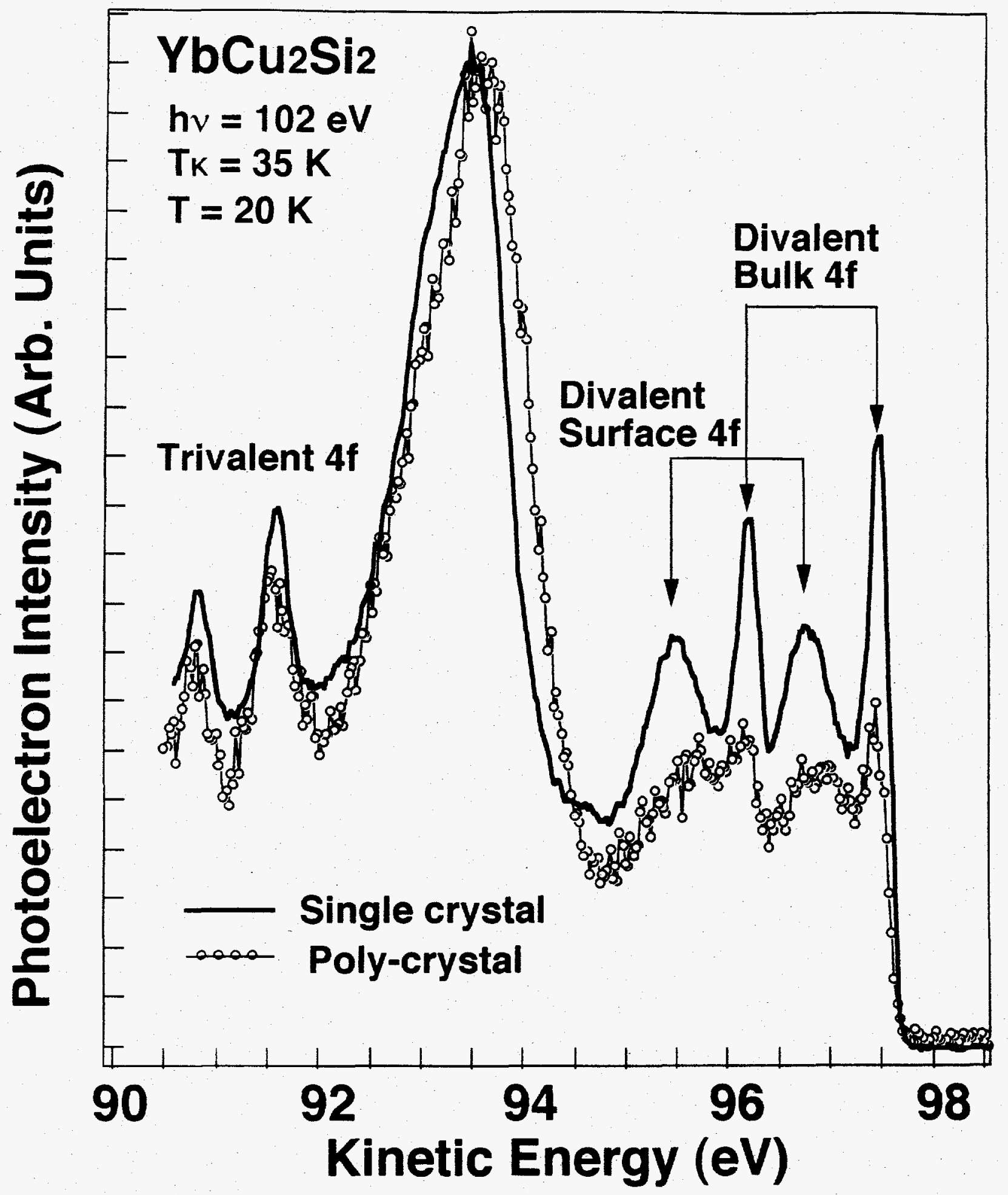

Fig. 3 


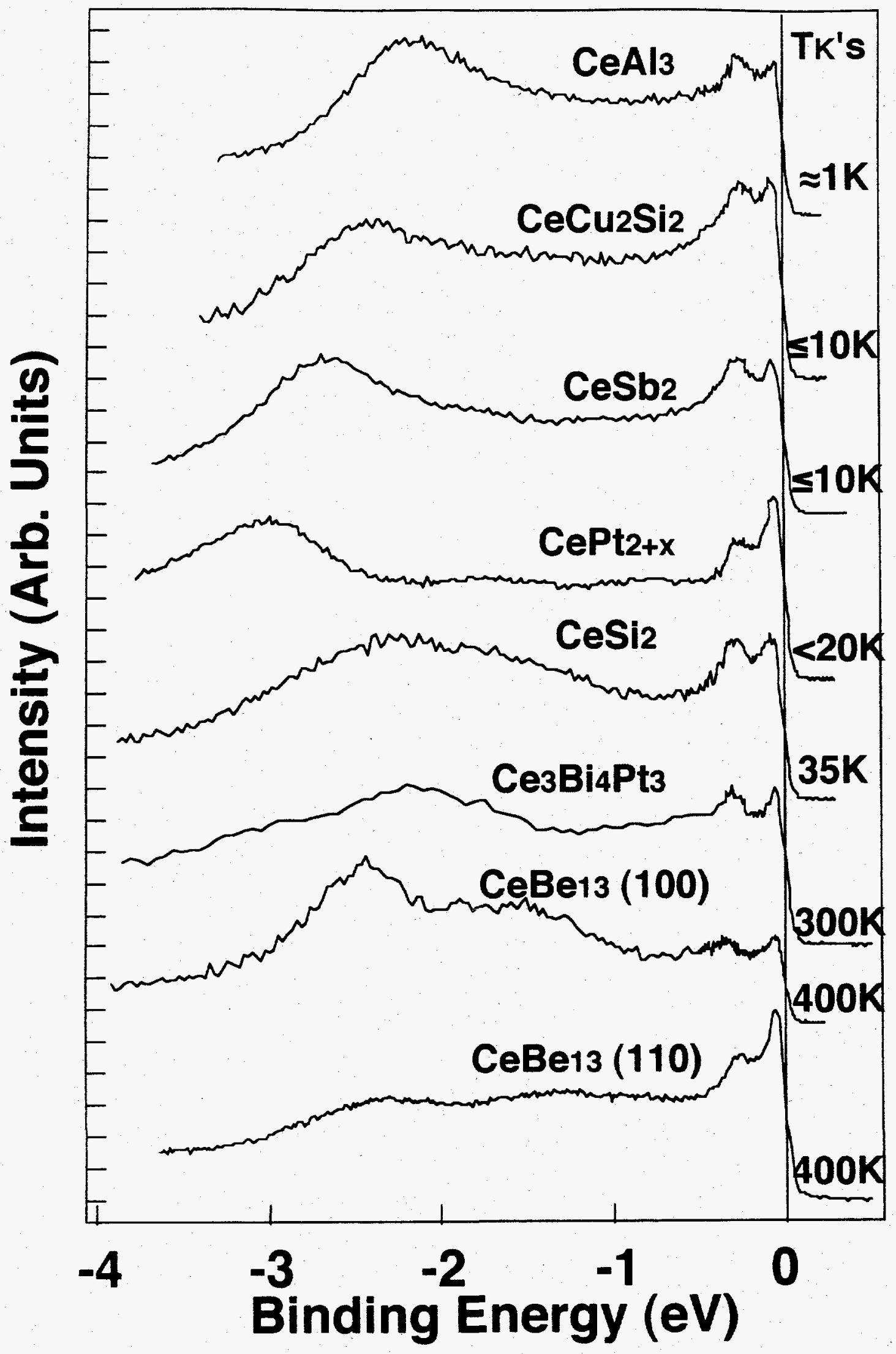




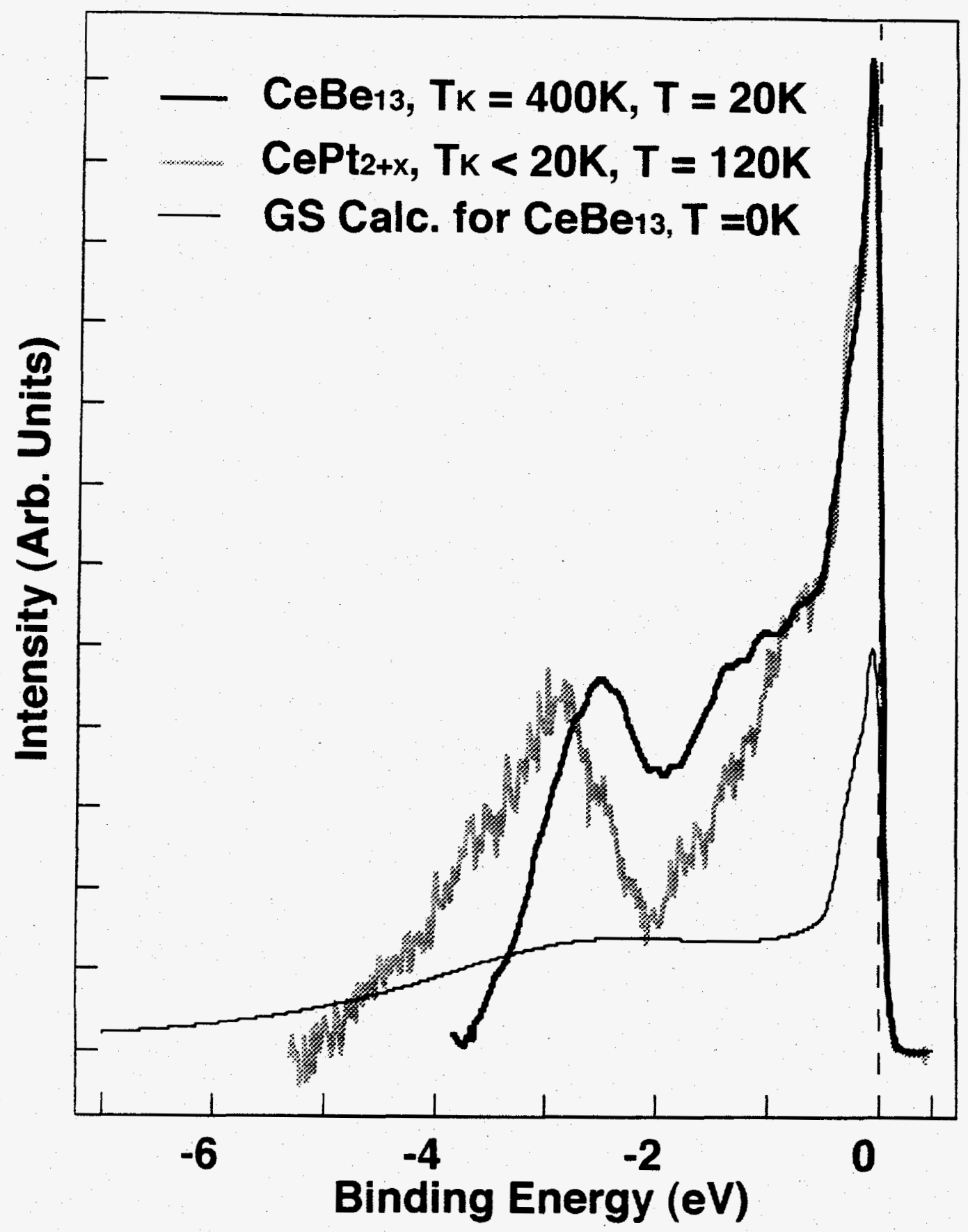

Fig.s 


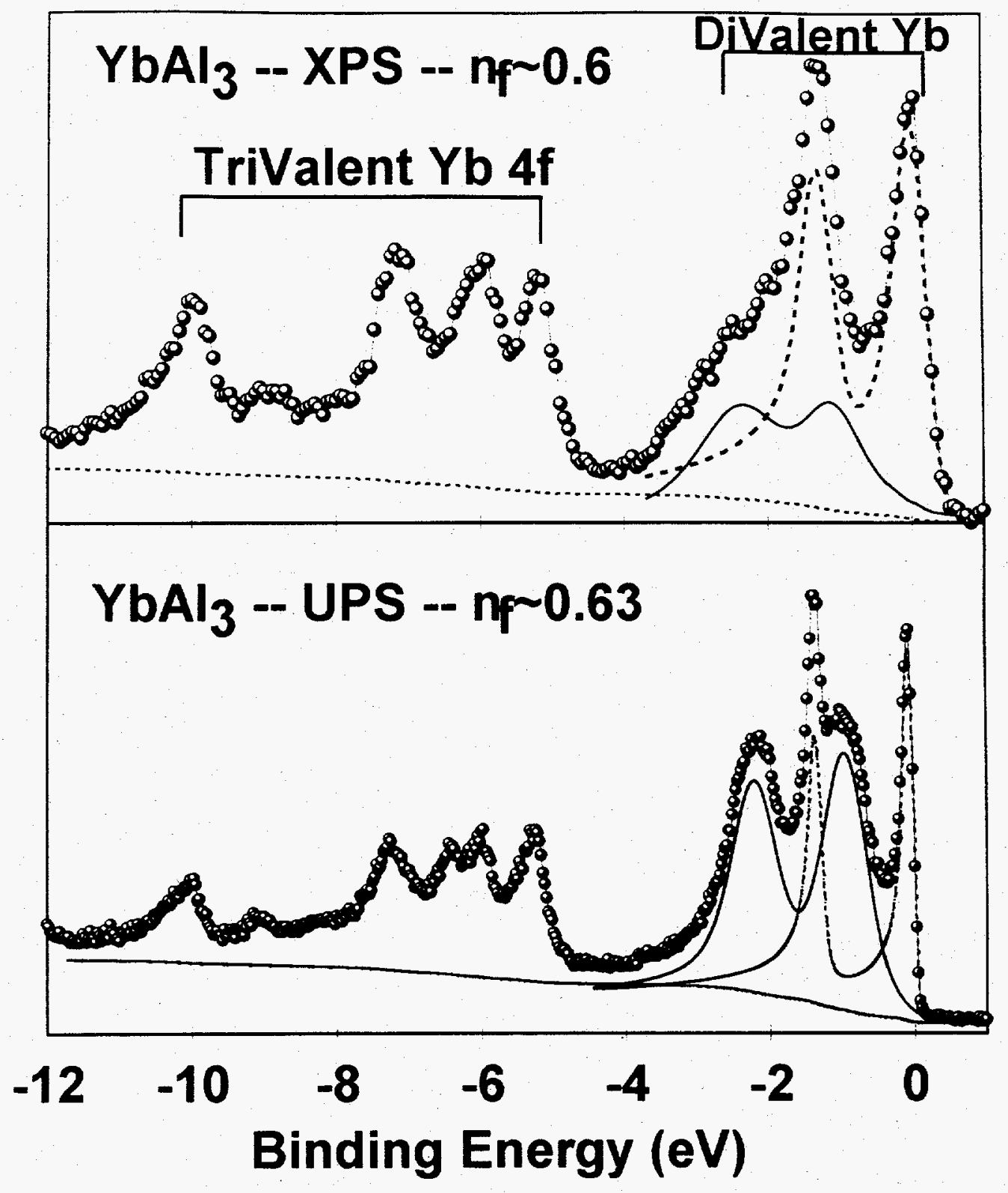

Fig. 6 


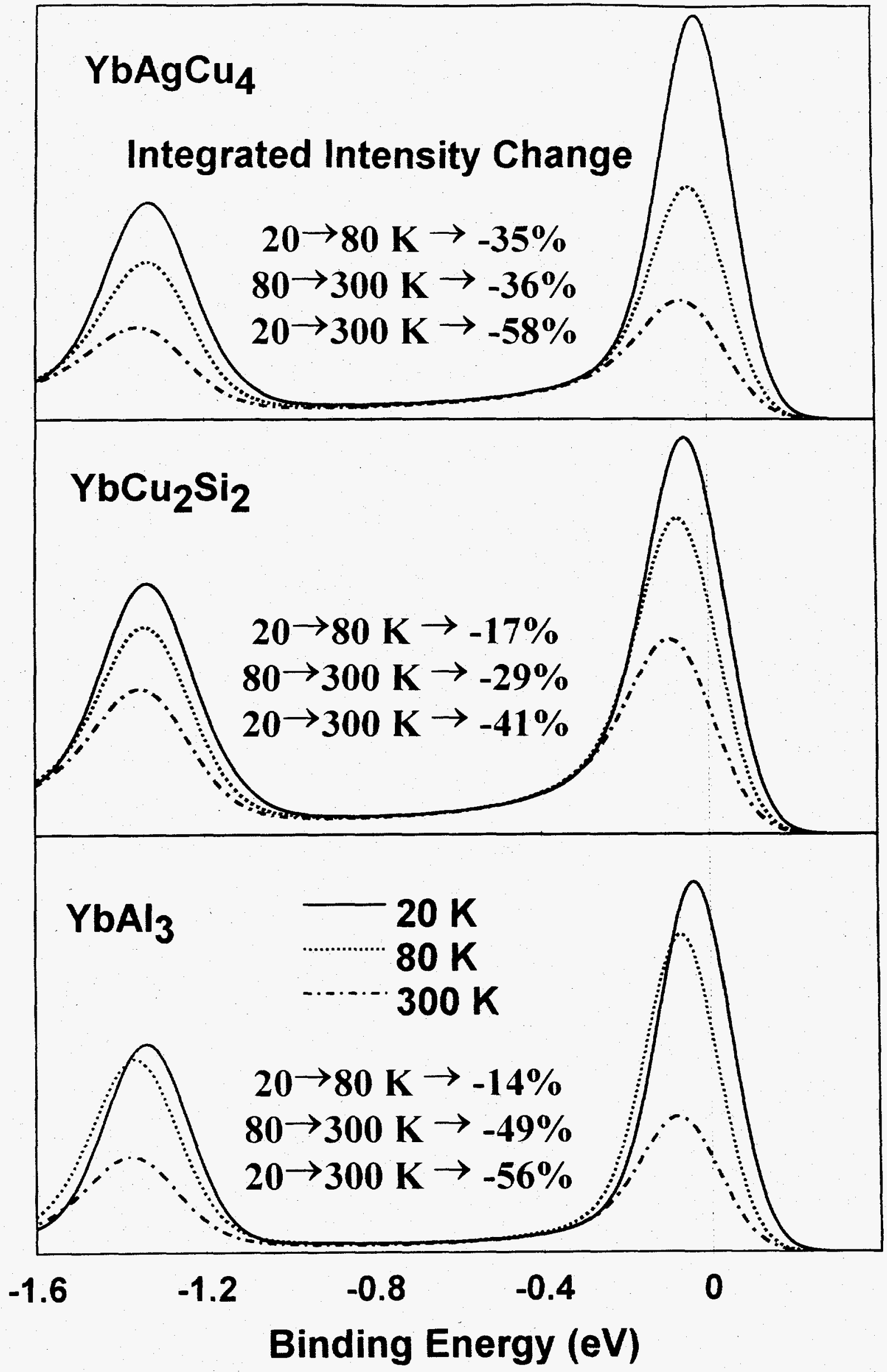

Fig. 


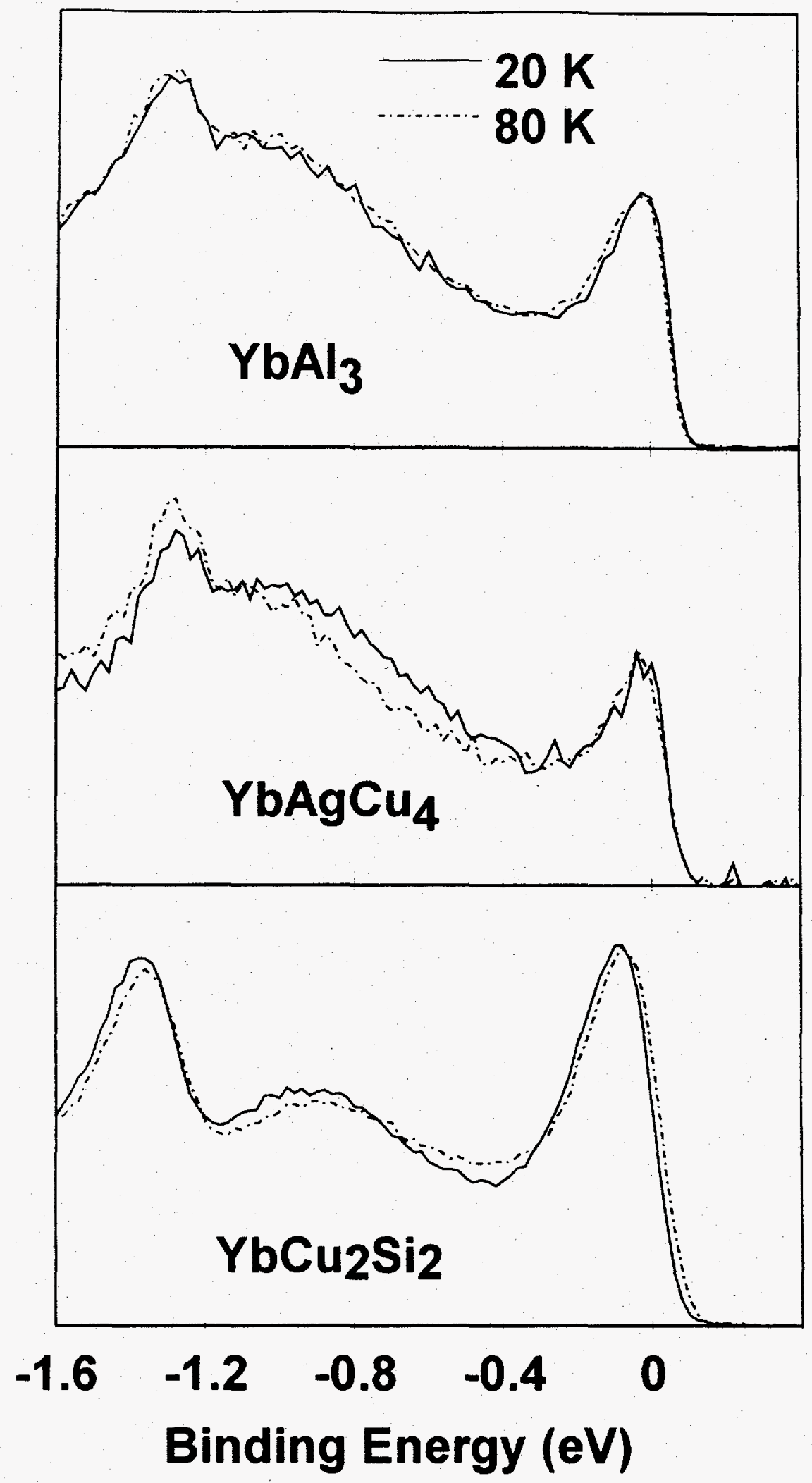

Fig. 8 


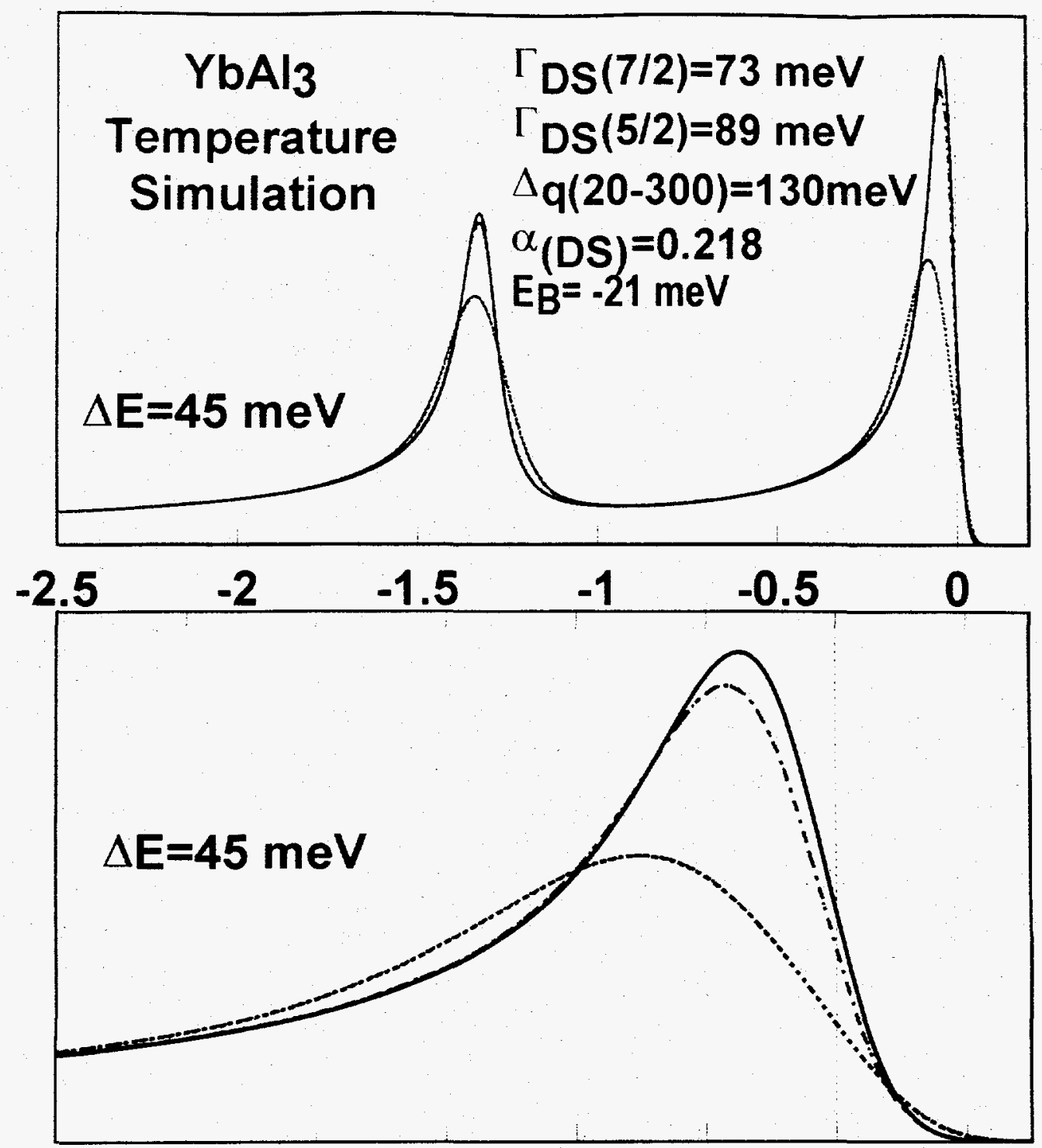

\begin{tabular}{llllllll}
-0.3 & -0.25 & -0.2 & -0.15 & -0.1 & -0.05 & 0 & 0.05 \\
\hline
\end{tabular}

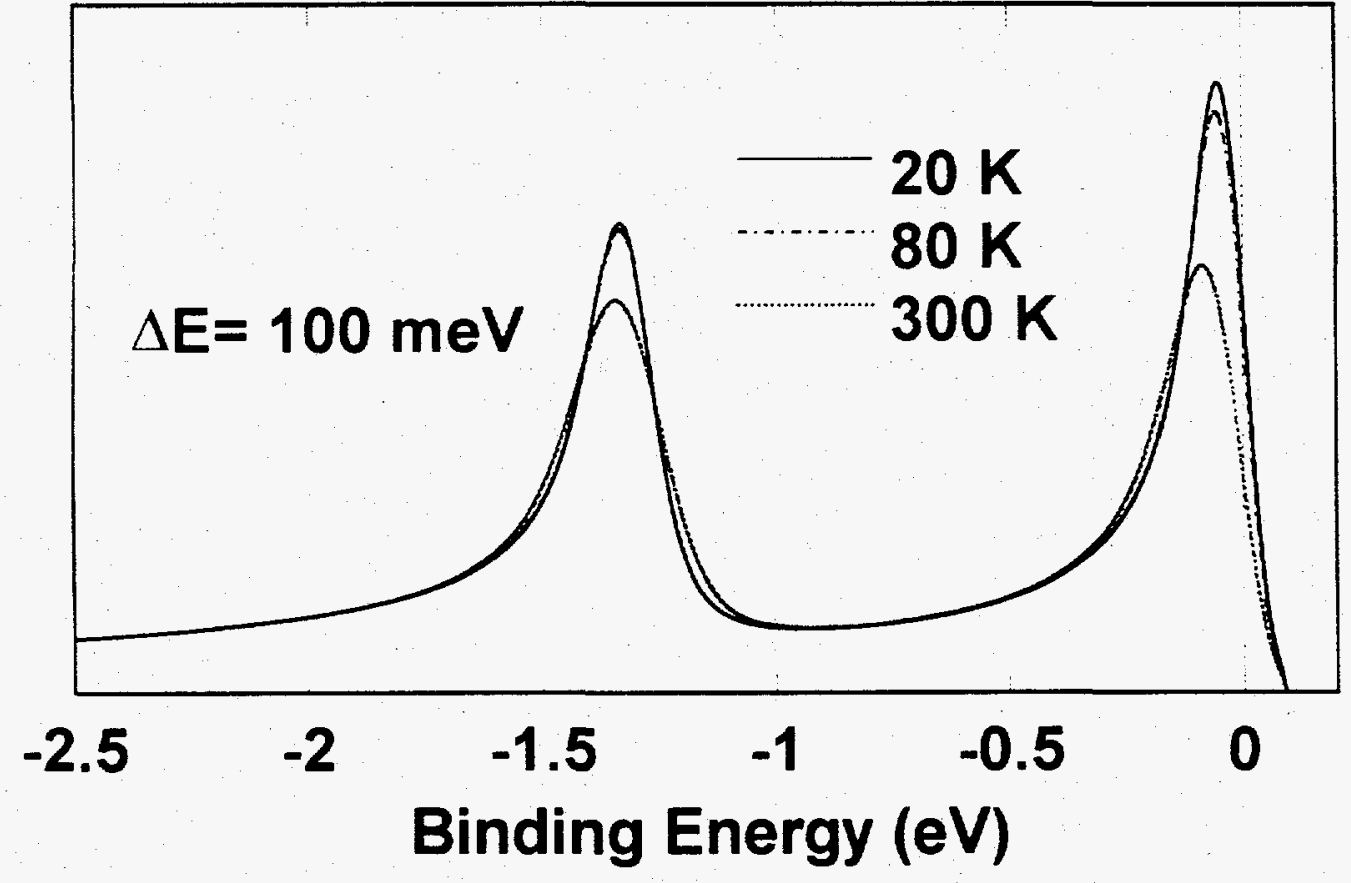




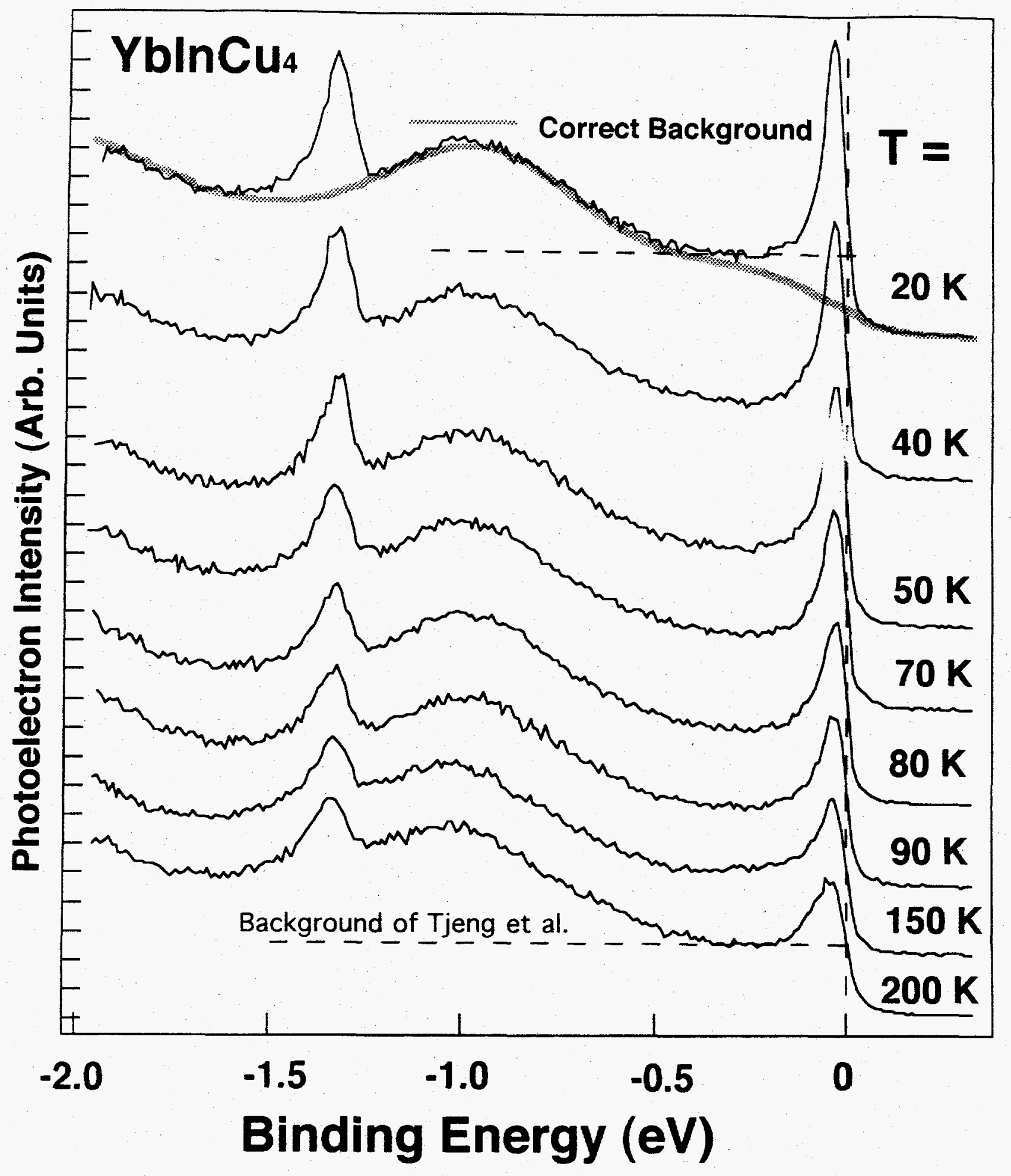

Fid. 


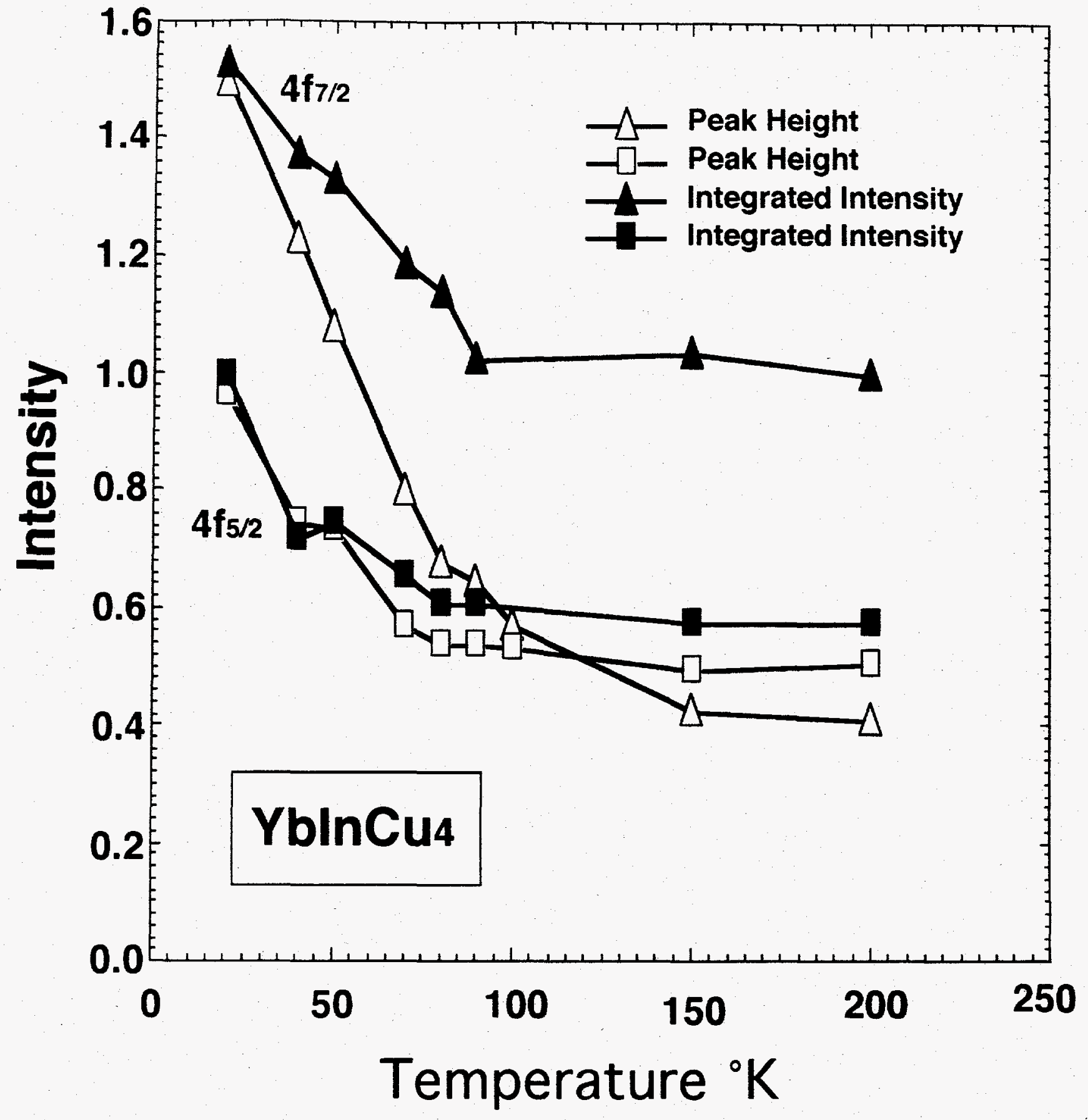

Fig."I 


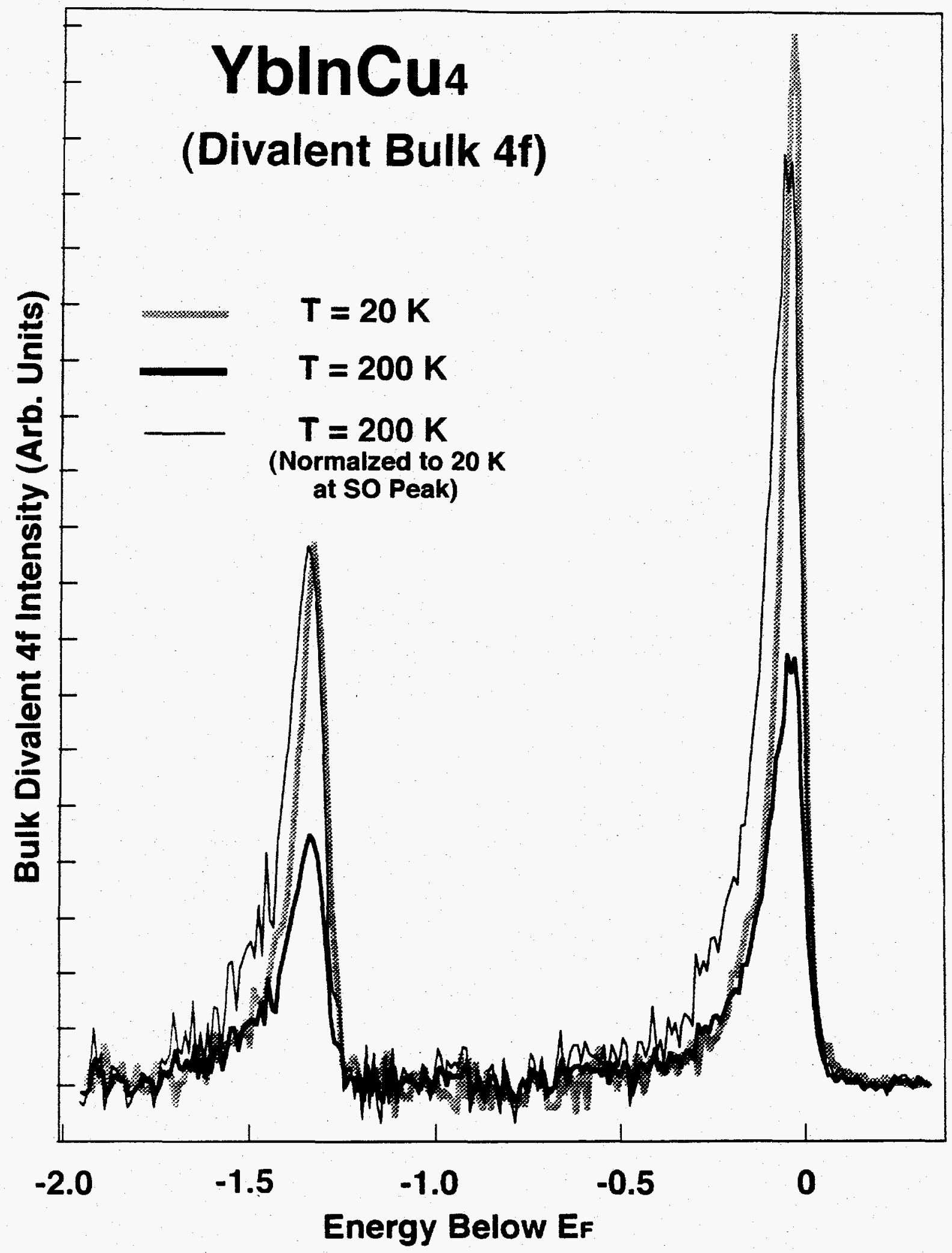

Fig. 12 


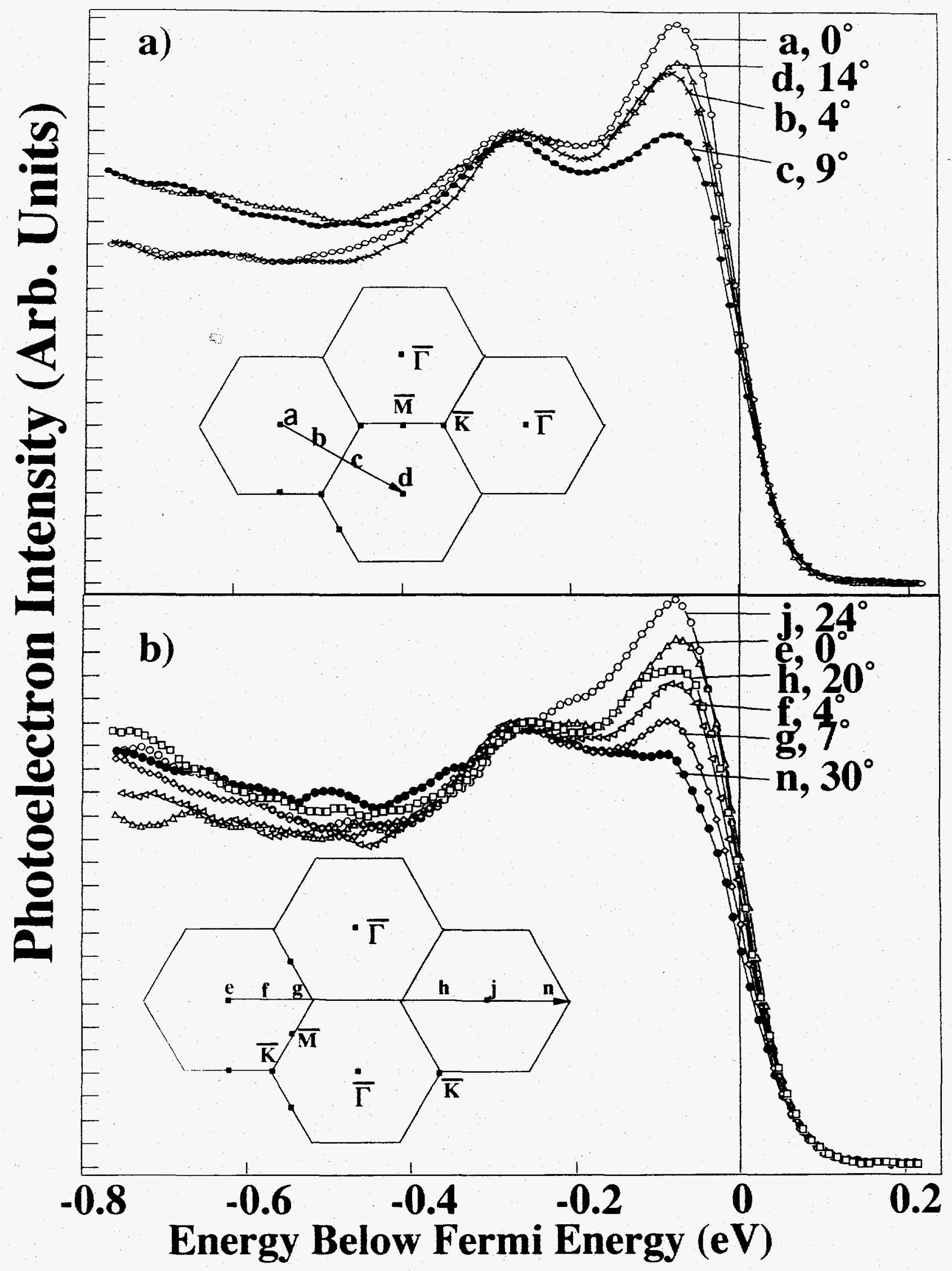

Fig. 13 
Photoelectron Intensity (Arb. Units)
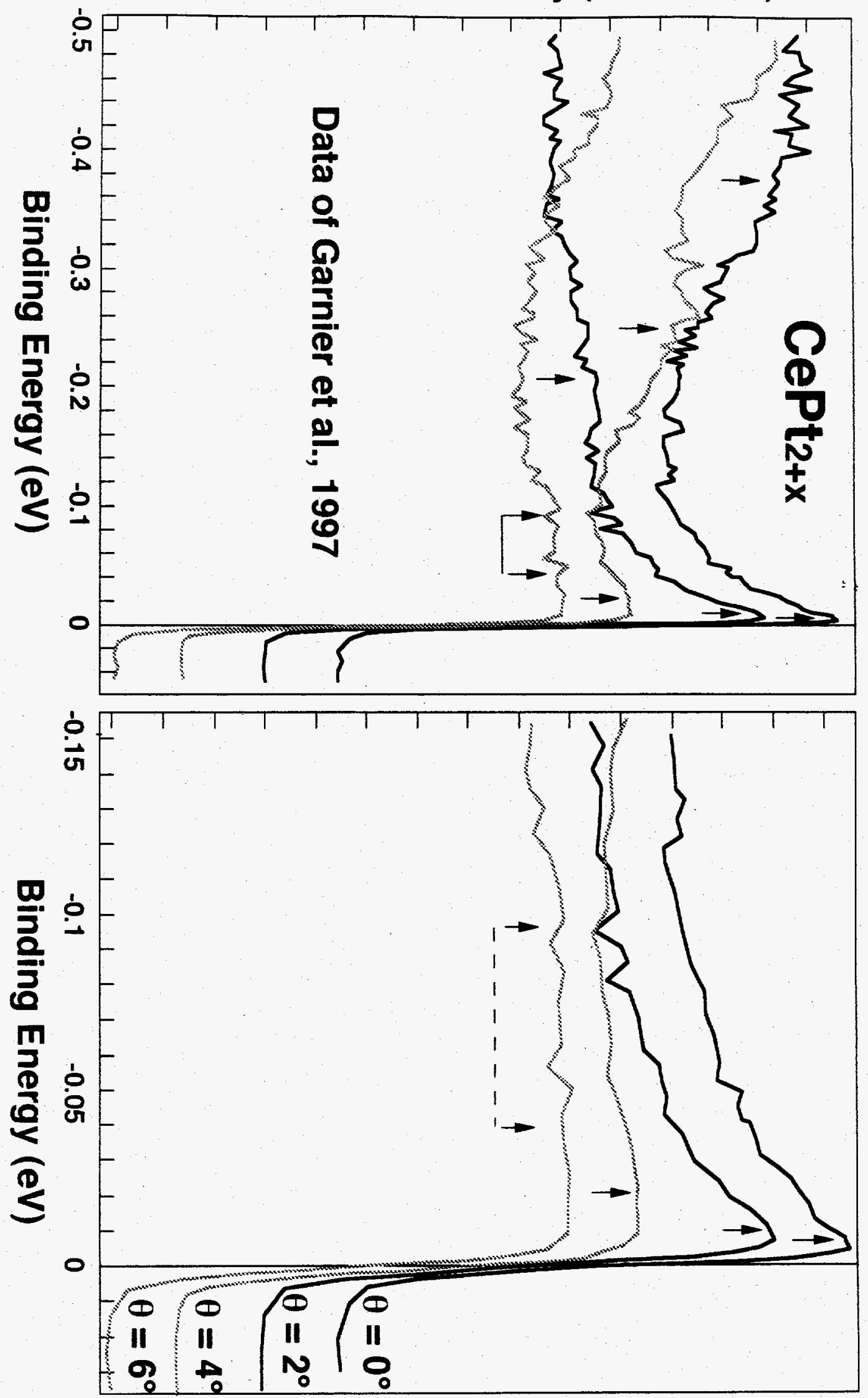

Fig. 14 
Photoelectron Intensity (Arb. Units)

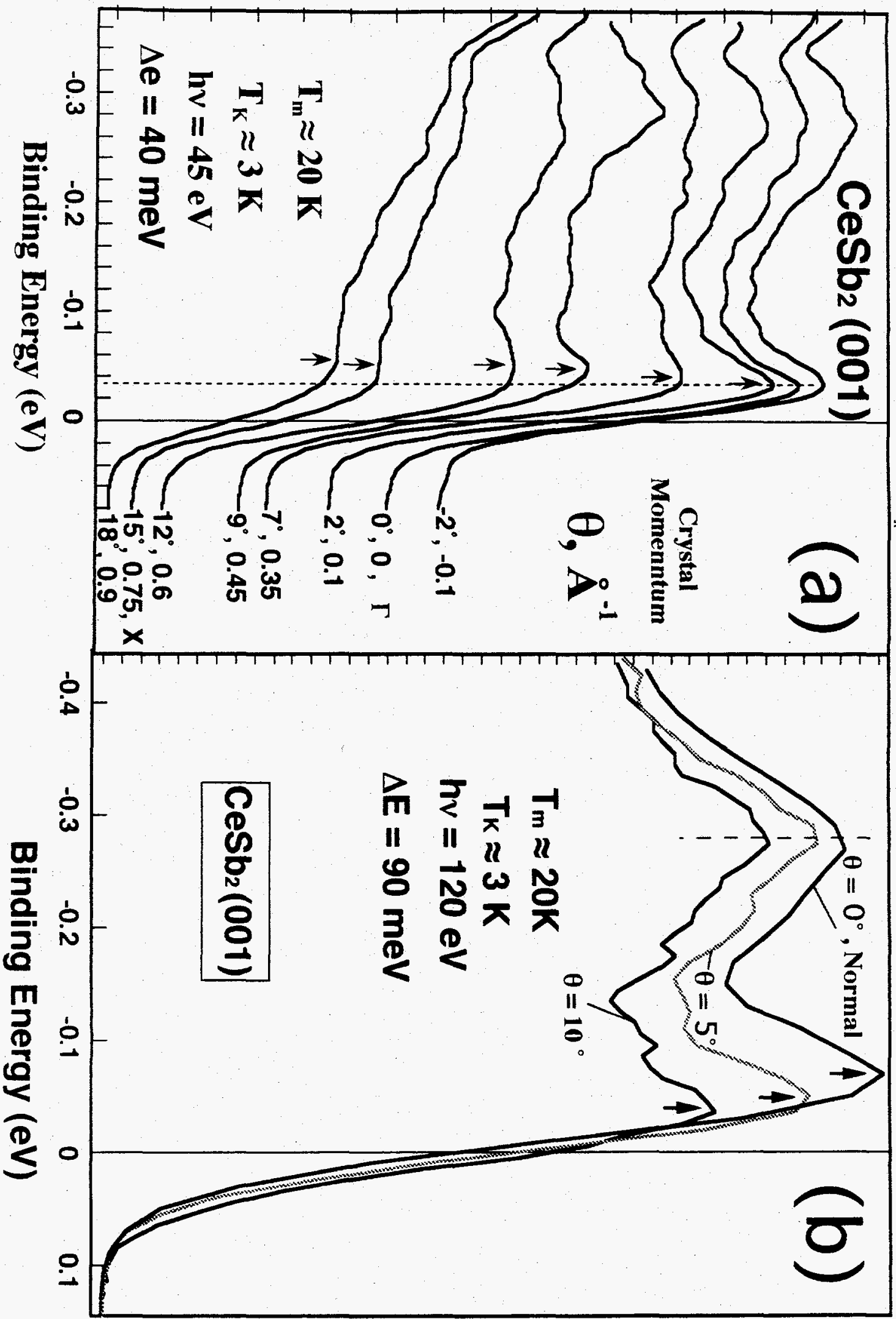

$F: Q, 15$ 


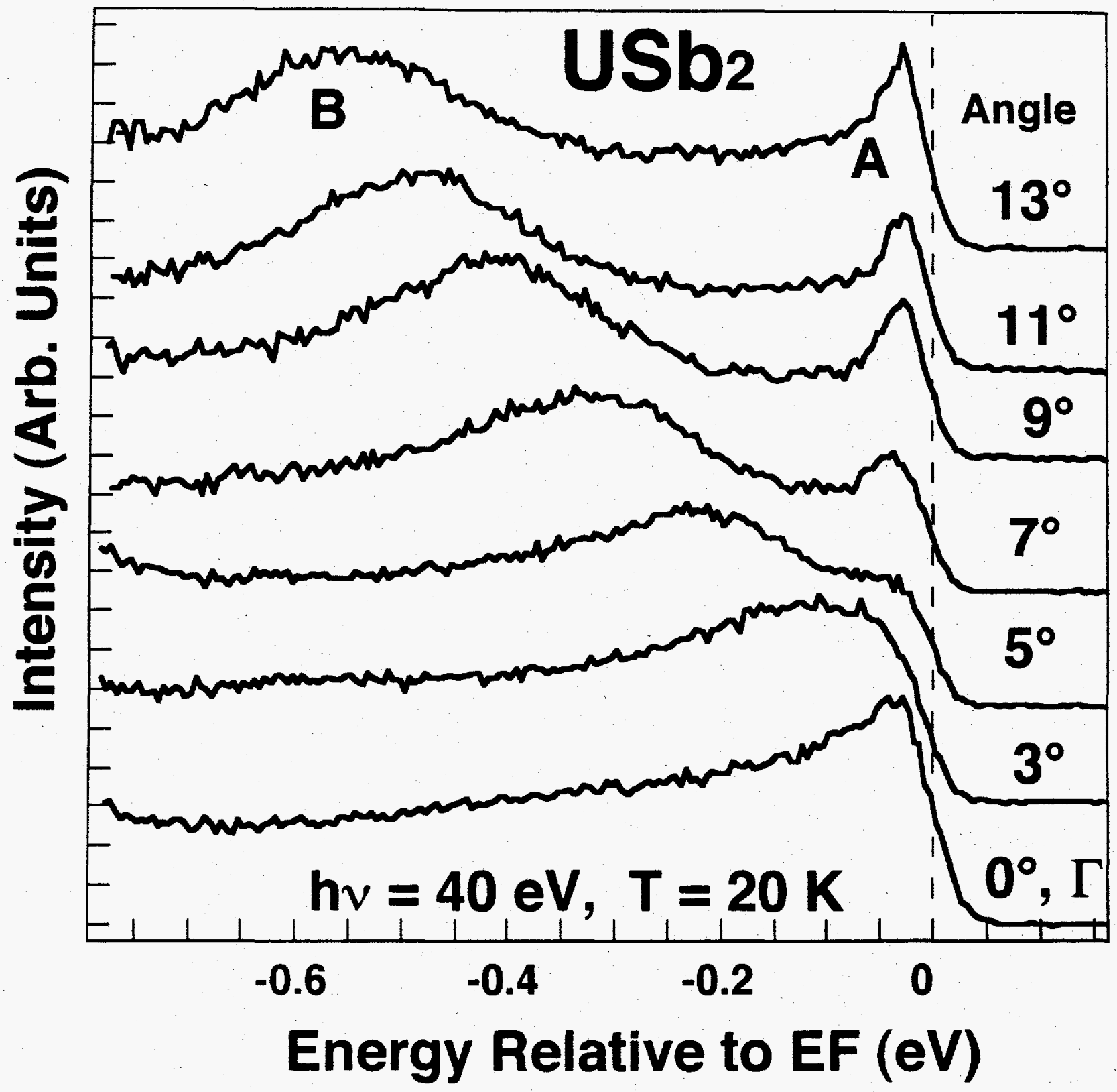

Fig. 16 


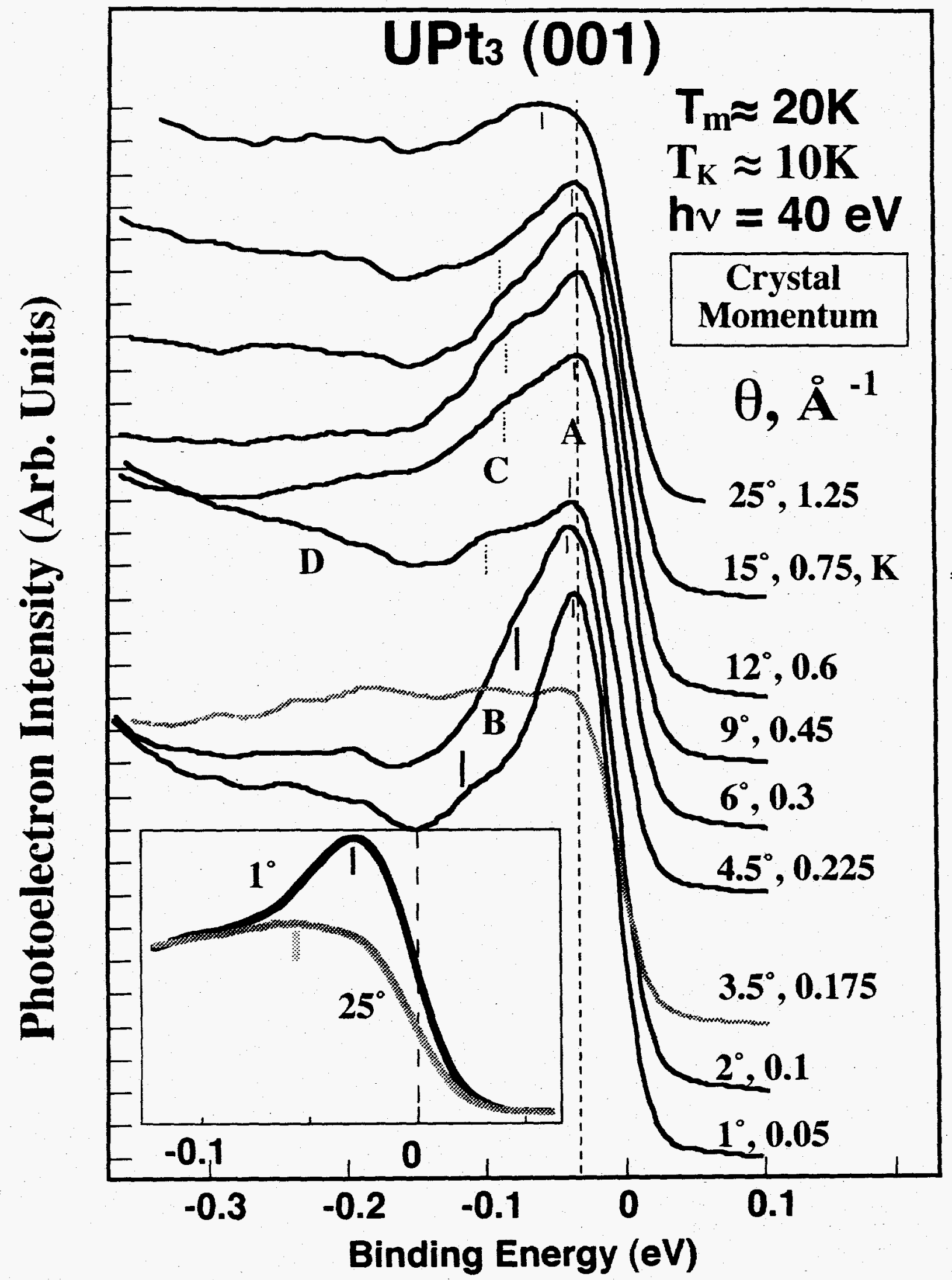

$\left.L^{-} ; g, 1\right\rangle$ 